\title{
Distributed State Estimation for Discrete-Time Sensor Networks with Randomly Varying Nonlinearities and Missing Measurements
}

\author{
Jinling Liang, Zidong Wang, Senior Member, IEEE, and Xiaohui Liu
}

\begin{abstract}
This paper deals with the distributed state estimation problem for a class of sensor networks described by discrete-time stochastic systems with randomly varying nonlinearities and missing measurements. In the sensor network, there is no centralized processor capable of collecting all the measurements from the sensors, and therefore each individual sensor needs to estimate the system state based not only on its own measurement but also on its neighboring sensors' measurements according to certain topology. The stochastic Brownian motions affect both the dynamical plant and the sensor measurement outputs. The randomly varying nonlinearities and missing measurements are introduced to reflect more realistic dynamical behaviors of the sensor networks that are caused by noisy environment as well as by probabilistic communication failures. Through available output measurements from each individual sensor, we aim to design distributed state estimators to approximate the states of the networked dynamic system. Sufficient conditions are presented to guarantee the convergence of the estimation error systems for all admissible stochastic disturbances, randomly varying nonlinearities, and missing measurements. Then, the explicit expressions of individual estimators are derived to facilitate the distributed computing of state estimation from each sensor. Finally, a numerical example is given to verify the theoretical results.
\end{abstract}

Index Terms-Distributed state estimation, missing measurements, randomly varying nonlinearity, sensor network, stochastic disturbances.

\section{INTRODUCTION}

D YNAMICS analysis for complex networks has long been a hot research topic that has been attracting recurring attention from a variety of research communities. The main reason lies in the fact that complex networks serve as natural models for many practical systems such as neuronal networks, genetic networks, networks in social sciences, power grid networks, coupled mechanical systems, information networks,

Manuscript received September 7, 2010; accepted December 16, 2010. Date of publication February 22, 2011; date of current version March 2, 2011. This work was supported in part by the Royal Society of U.K., the National Natural Science Foundation of China under Grant 60804028 and Grant 61028008, the Teaching and Research Fund for Excellent Young Teachers at Southeast University of China, the Qing Lan Project of Jiangsu Province of China, the International Science and Technology Cooperation Project of China under Grant 2009DFA32050, and the Alexander von Humboldt Foundation of Germany.

J. Liang is with the Department of Mathematics, Southeast University, Nanjing 210096, China (e-mail: jinlliang@gmail.com).

Z. Wang and X. Liu are with the Department of Information Systems and Computing, Brunel University, Uxbridge UB8 3PH, U.K. (e-mail: Zidong.Wang@brunel.ac.uk; Xiaohui.Liu@brunel.ac.uk).

Digital Object Identifier 10.1109/TNN.2011.2105501 and others. In a complex network, the connection weights of the nodes are largely dependent on certain resistance and capacitance values, which include uncertainties (modeling errors) subject to stochastic disturbances as well as limited communication constraints. Therefore, complex networks exhibit grand challenges for the understanding of stochastic influence, network-induced phenomena, cluster formation, stability, sensitivity and robustness, bifurcations and chaos, etc. In the past decade, a rich body of literature has appeared on the general topic of complex networks and applications, see [1]-[7] and the references therein.

Sensor networks, as a special class of complex networks, have recently been undergoing a quiet revolution in all aspects of the hardware implementation, software development, and theoretical research. In addition to the universal attributes of complex networks, sensor networks do possess their own characteristics due mainly to the large number of inexpensive wireless devices (nodes) densely distributed over the region of interest [8], [9]. As discussed in [8], two interconnected critical challenges for sensor networks are the efficient networking techniques and the collaborative signal processing (CSP) to efficiently process the distributed information gathered. Lying in the core part of the area of CSP is the distributed filtering or estimation problem, which has recently been attracting growing research interests. For distributed estimation problem, the inherently asynchronous sensor network is comprised of a large number of sensor nodes with computing and wireless communication capabilities, where the nodes are spatially distributed to form a wireless ad hoc network and every node has its own notion of time. Each individual sensor in a sensor network locally estimates the system state from not only its own measurement but also its neighboring sensors' measurements according to the given topology.

Different from the traditional central filtering techniques [10]-[15], an effective distributed estimation algorithm should be capable of handling two additional issues: 1) complicated coupling between the sensor nodes according to a given topology, and 2) network-induced phenomena such as randomly varying nonlinearities and missing measurements. Concerning the node coupling issue, four arguably representative distributed strategies have been proposed in the recent literature: 1) the distributed Kalman filtering algorithm [16]-[21] with different sensing models and different consensus strategies; 2) the distributed estimation algorithm [22]-[24] that adaptively updates the weights for minimizing the estimation error 
variance; 3) the multiscale approach [25] to accelerate the convergence of decentralized consensus problems for networks with poor connectivity; and 4) the distributed diffusion filtering algorithm [26], [27] with the information diffused across the network through a sequence of Kalman iterations and data aggregation. However, compared to the fruitful results with respect to sensor node coupling up to now, the corresponding research for distributed estimation problems with random communication constraints has received much less attention, and this constitutes the main motivation for the present research.

In a sensor network, each sensor node has wireless communication capability as well as some level of intelligence for signal processing and disseminating data. These sensor nodes collaborate among themselves to set up a sensing network. However, the limited energy, computational power, and communication resources of the sensor nodes will inevitably lead to communication constraints that pose great challenges in designing distributed estimators. Firstly, the sensor measurements are usually subject to probabilistic information, missing which may be caused for a variety of reasons such as the high maneuverability of the tracked target, intermittent sensor failures, or limited battery energy. Note that such a missing measurement phenomenon typically occurs in networked control systems and has attracted considerable attention during the past few years, see [28]-[30]. Secondly, the sensor networks are often influenced by additive nonlinear disturbances that are caused by environmental circumstances. Such nonlinear disturbances themselves may experience random abrupt changes due probably to abrupt phenomena such as random failures and changes in node interconnections, which give rise to the so-called randomly varying nonlinearities, see [31], [32] and [33] for more explanations. Thirdly, stochastic disturbances are unavoidable when modeling sensor networks in a noisy environment. Note that the distributed estimation problem has been extensively studied for sensor networks with additive white noises in [19], [24], [26] and with multiplicative (Itô-type) noises in [34]. Unfortunately, so far very little research effort has been made to the network-induced random phenomena including multiplicative noises, random measurement missing, as well as randomly varying nonlinearities. Therefore, the main purpose of this paper is to pave the way for dealing with distributed estimation problems with random communication constraints.

In this paper, attention is focused on the distributed state estimation problem for a class of sensor networks described by discrete-time stochastic systems with randomly varying nonlinearities and missing measurements. The main contributions of this paper lie in the new research problem and can be summarized as follows. 1) The randomly varying nonlinearities are introduced to describe the binary switch between two kinds of nonlinear disturbances governed by a Bernoulli-distributed white noise sequence. 2) The sensor model includes the probabilistic missing measurements that account for the random packet dropout during the signal transmission or information collection amongst the sensor network. 3) Intensive stochastic analysis is carried out in order to establish a unified framework that caters the simultaneous presence of the randomly varying nonlinearities, the probabilistic missing measurements, and the multiplicative noises. In Section II, the problem addressed is formulated and some preliminaries are briefly outlined. In Section III, the main theorems and corollaries are given for the distributed state estimation of the dynamical systems describing the sensor networks. In Section IV, a numerical example is given to demonstrate the effectiveness of the distributed state estimation scheme. Finally, conclusions are drawn in Section V.

\section{Preliminaries and Problem Statement}

Notations: Throughout this paper, $I$ and 0 represent, respectively, the identity matrix and the zero matrix of appropriate dimensions. $\mathbb{N}$ is used to be the set $\{1,2, \ldots\}$. The Kronecker product of matrices $A \in \mathbb{R}^{m \times n}$ and $B \in \mathbb{R}^{p \times q}$ is a matrix in $\mathbb{R}^{m p \times n q}$ and denoted as $A \otimes B$. The notation $X>0$ for $X \in \mathbb{R}^{n \times n}$ means that matrix $X$ is real, symmetric, and positive definite, while $\operatorname{Sym}(X)$ represents the matrix $X+X^{T}$. $\operatorname{diag}(\cdots)$ and $\operatorname{col}(\cdots)$ stand for, respectively, the blockdiagonal matrix and the matrix column with blocks given by the matrices in $(\cdots) .(\Omega, \mathcal{F}, \mathscr{P})$ is a complete probability space with the probability measure $\mathscr{P}$ having total mass 1 . $\operatorname{Prob}\{\beta\}$ stands for the occurrence probability of the event $\beta$ and $\mathbb{E}\left\{\alpha_{1}\right\}, \mathbb{E}\left\{\alpha_{1} \mid \alpha_{2}\right\}$ mean, respectively, the mathematical expectation of the stochastic variable $\alpha_{1}$ and the expectation of $\alpha_{1}$ conditional on $\alpha_{2}$ with respect to the given probability measure $\mathscr{P}$. $\|\cdot\|$ refers to the Euclidean vector norm and the asterisk "*" in a symmetric matrix is used to denote the term that is induced by symmetry. Matrices, if not stated, are assumed to be compatible for algebraic operations.

It is assumed in this paper that the sensor network has $N$ sensor nodes which are distributed in the space according to a specific interconnection topology characterized by a directed graph $\mathcal{G}=(\mathcal{V}, \mathcal{E}, L)$, where $\mathcal{V}=\{1,2, \ldots, N\}$ denotes the set of sensor nodes, $\mathcal{E} \subseteq \mathcal{V} \times \mathcal{V}$ is the set of edges, and $L=$ $\left(l_{i j}\right)_{N \times N}$ is the nonnegative adjacency matrix associated with the edges of the graph, i.e., $l_{i j}>0 \Leftrightarrow$ edge $(i, j) \in \mathcal{E}$, which means that there is information transmission from sensor $j$ to sensor $i$. Moreover, it is also assumed that $l_{i i}=1$ for all $i \in \mathcal{V}$, i.e., the sensors are self-connected. If $(i, j) \in \mathcal{E}$, then node $j$ is called one of the neighbors of node $i$. For all $i \in \mathcal{V}$, denote $\mathcal{N}_{i} \triangleq\{j \in \mathcal{V} \mid(i, j) \in \mathcal{E}\}$, which means that in the sensor network, sensor node $i$ can receive the information from its neighboring nodes $j \in \mathcal{N}_{i}$ according to the given network topology.

A target plant is the system whose state is to be estimated through the distributed sensors. Let the target plant be described by the following discrete-time nonlinear stochastic system defined on the complete probability space $(\Omega, \mathcal{F}, \mathscr{P})$ :

$$
\begin{aligned}
x(k+1)= & A x(k)+D x(k-\tau(k))+\delta(k) B_{1} f(k, x(k)) \\
& +(1-\delta(k)) B_{2} g(k, x(k))+M_{0} x(k) \omega_{0}(k)
\end{aligned}
$$

with $N$ sensors modeled by

$$
y_{i}(k)=\gamma_{i}(k) C_{i} x(k)+M_{i} x(k) \omega_{i}(k), \quad i=1,2, \ldots, N
$$

where $k \in \mathbb{N}$ and $x(k) \in \mathbb{R}^{n}$ is the state of the target; $y_{i}(k) \in$ $\mathbb{R}^{m}$ is the measurement output measured by sensor $i$ on the target $x(k) ; A, D, B_{1}, B_{2}, M_{0}, C_{i}$, and $M_{i}$ are known constant 
matrices; $f(\cdot, \cdot): \mathbb{N} \times \mathbb{R}^{n} \rightarrow \mathbb{R}^{n}$ and $g(\cdot, \cdot): \mathbb{N} \times \mathbb{R}^{n} \rightarrow \mathbb{R}^{n}$ are nonlinear functions; the time-varying integer $\tau(k)$ corresponds to the delay in the state dynamics and satisfies $\tau_{1} \leq \tau(k) \leq \tau_{2}$ ( $\tau_{1}$ and $\tau_{2}$ are known positive integers); $\omega_{0}(k)$ and $\omega_{i}(k)(i=$ $1,2, \ldots, N)$ are scalar Wiener processes (Brownian motions) defined on the complete probability space $(\Omega, \mathcal{F}, \mathscr{P})$ with

$$
\begin{aligned}
& \mathbb{E}\left\{\omega_{i}(k)\right\}=0 \quad \mathbb{E}\left\{\omega_{i}^{2}(k)\right\}=1 ; \\
& \mathbb{E}\left\{\omega_{i}(s) \omega_{i}(t)\right\}=0(s \neq t \in \mathbb{N}) ; \quad i=0,1,2, \ldots, N .
\end{aligned}
$$

The stochastic variables $\delta(k) \in \mathbb{R}$ and $\gamma_{i}(k) \in \mathbb{R}(i=$ $1,2, \ldots, N)$ are Bernoulli-distributed white noise sequences specified by the following distribution laws:

$$
\begin{aligned}
& \operatorname{Prob}\{\delta(k)=1\}=\mathbb{E}\{\delta(k)\}=\bar{\delta} \\
& \operatorname{Prob}\{\delta(k)=0\}=1-\mathbb{E}\{\delta(k)\}=1-\bar{\delta} \\
& \operatorname{Prob}\left\{\gamma_{i}(k)=1\right\}=\mathbb{E}\left\{\gamma_{i}(k)\right\}=\bar{\gamma}_{i} \\
& \operatorname{Prob}\left\{\gamma_{i}(k)=0\right\}=1-\mathbb{E}\left\{\gamma_{i}(k)\right\}=1-\bar{\gamma}_{i}
\end{aligned}
$$

where $\bar{\delta}, \bar{\gamma}_{i} \in[0,1]$ are known constants. Obviously, for the stochastic variables $\delta(k)$ and $\gamma_{i}(k)$, one has

$\mathbb{E}\left\{(\delta(k)-\bar{\delta})^{2}\right\}=\bar{\delta}(1-\bar{\delta}), \mathbb{E}\left\{\left(\gamma_{i}(k)-\bar{\gamma}_{i}\right)^{2}\right\}=\bar{\gamma}_{i}\left(1-\bar{\gamma}_{i}\right)$.

Here, it is further assumed that the variables $\delta(k), \gamma_{i}(k)(i=$ $1,2, \ldots, N)$, and $\omega_{j}(k)(j=0,1, \ldots, N)$ are mutually independent.

Remark 1: Due to the random abrupt changes in the environmental circumstances such as repairs of the components, random failures, and changes in the interconnections of subsystems, the nonlinear disturbances may occur in a probabilistic way and are randomly changeable in terms of their types or intensity. In the dynamic target model (1), the random variable $\delta(k)$ is used to model the probability distribution of the nonlinear functions. In other words, in the target model (1), the two terms $\delta(k) B_{1} f(k, x(k))$ and $(1-\delta(k)) B_{2} g(k, x(k))$ can be used to account for the binary switches between these two nonlinear functions according to a given probability distribution. Such a novel idea was first proposed in [31] and [32] to investigate the synchronization problem of stochastic delayed complex networks. The randomly varying nonlinearities, also called stochastic nonlinearities, have recently received some research interests in the literature. For example, in [35], the control problem for discrete-time systems with stochastic nonlinearities has been thoroughly investigated.

Remark 2: Missing measurements are considered in the sensor network (2) by resorting to the random variable $\gamma_{i}(k)$. Note that such system measurement mode (2) and (5) was first introduced in [36] and subsequently utilized in many other papers, see [28]-[30]. The case of $\gamma_{i}(k)=0$, which does happen in practice, implies that the main signal is missing and the system measurement contains state-dependent noises only.

As stated in the introduction, the aim of this paper is to design state estimators to approximate the states of the networked dynamical target on the condition that there is no centralized processor capable of collecting all the measurements from the sensors. By considering the neighboring measurements in current times, here we construct the following distributed state estimator to track the state $x(k)$ of the target:

$$
\begin{aligned}
\hat{x}_{i}(k+1)= & A \hat{x}_{i}(k)+\bar{\delta} B_{1} f\left(k, \hat{x}_{i}(k)\right) \\
& +(1-\bar{\delta}) B_{2} g\left(k, \hat{x}_{i}(k)\right) \\
& +\sum_{j \in \mathcal{N}_{i}} l_{i j} K_{i j}\left(y_{j}(k)-\bar{\gamma}_{j} C_{j} \hat{x}_{j}(k)\right)
\end{aligned}
$$

where $k \in \mathbb{N} ; i=1,2, \ldots, N ; \hat{x}_{i}(k)$ is the estimation of the networked system state $x(k)$ on sensor $i$ and $K_{i j} \in \mathbb{R}^{n \times m}$ is the estimator gain matrix to be determined.

Throughout this paper, we assume that the target plant is observable and the following conditions hold for the nonlinear functions $f$ and $g$ in model (1):

Assumption 1: $f(k, 0)=0$ and $g(k, 0)=0$ for all $k \in \mathbb{N}$.

Assumption 2: There exist matrices $\Sigma_{1}$ and $\Sigma_{2}$ such that the following inequalities hold:

$$
\begin{aligned}
\|f(k, u)-f(k, v)\| & \leq\left\|\Sigma_{1}(u-v)\right\| \\
\|g(k, u)-g(k, v)\| & \leq\left\|\Sigma_{2}(u-v)\right\|
\end{aligned}
$$

for all $k \in \mathbb{N}$ and $u, v \in \mathbb{R}^{n}$.

Setting $\tilde{x}_{i}(k)=x(k)-\hat{x}_{i}(k)$, the estimation error dynamics for sensor $i$ can be obtained from (1), (2), and (6) as follows:

$$
\begin{aligned}
\tilde{x}_{i}(k+1)= & A \tilde{x}_{i}(k)+D x(k-\tau(k))+\bar{\delta} B_{1} \tilde{f}\left(k, \tilde{x}_{i}(k)\right) \\
& +(1-\bar{\delta}) B_{2} \tilde{g}\left(k, \tilde{x}_{i}(k)\right)+M_{0} x(k) \omega_{0}(k) \\
& +(\delta(k)-\bar{\delta})\left(B_{1} f(k, x(k))-B_{2} g(k, x(k))\right) \\
& -\sum_{j \in \mathcal{N}_{i}} l_{i j} K_{i j}\left(\bar{\gamma}_{j} C_{j} \tilde{x}_{j}(k)+M_{j} x(k) \omega_{j}(k)\right. \\
& \left.+\left(\gamma_{j}(k)-\bar{\gamma}_{j}\right) C_{j} x(k)\right)
\end{aligned}
$$

where $\tilde{f}\left(k, \tilde{x}_{i}(k)\right)=f(k, x(k))-f\left(k, \hat{x}_{i}(k)\right), \tilde{g}\left(k, \tilde{x}_{i}(k)\right)=$ $g(k, x(k))-g\left(k, \hat{x}_{i}(k)\right)$. By utilizing the Kronecker product, the error dynamics governed by (7) above can be rewritten in a compact form as

$$
\begin{aligned}
\tilde{x}(k+1)= & \left(I_{N} \otimes A-\bar{K} \Gamma \tilde{C}\right) \tilde{x}(k)+\tilde{D} x(k-\tau(k)) \\
& +(1-\bar{\delta})\left(I_{N} \otimes B_{2}\right) G(k, \tilde{x}(k)) \\
& +(\delta(k)-\bar{\delta})\left(\tilde{B}_{1} f(k, x(k))-\tilde{B}_{2} g(k, x(k))\right) \\
& +\tilde{M}_{0} x(k) \omega_{0}(k)+\bar{\delta}\left(I_{N} \otimes B_{1}\right) F(k, \tilde{x}(k)) \\
& -\bar{K}(\Gamma(k)-\Gamma) \bar{C} x(k)-\bar{K} \bar{\omega}(k) \tilde{M} x(k)
\end{aligned}
$$

where $\tilde{x}(k)=\left(\tilde{x}_{1}^{T}(k), \tilde{x}_{2}^{T}(k), \ldots, \tilde{x}_{N}^{T}(k)\right)^{T}, \tilde{C}=\operatorname{diag}\left(C_{1}\right.$, $\left.C_{2}, \ldots, C_{N}\right), \Gamma=\operatorname{diag}\left(\bar{\gamma}_{1} I_{m}, \bar{\gamma}_{2} I_{m}, \ldots, \bar{\gamma}_{N} I_{m}\right), \quad \Gamma(k)=$ $\operatorname{diag}\left(\gamma_{1}(k) I_{m}, \gamma_{2}(k) I_{m}, \ldots, \gamma_{N}(k) I_{m}\right), \bar{\omega}(k)=\operatorname{diag}\left(\omega_{1}(k) I_{m}\right.$, $\left.\omega_{2}(k) I_{m}, \ldots, \omega_{N}(k) I_{m}\right)$

$$
\begin{aligned}
& \tilde{B}_{1}=[\underbrace{B_{1}^{T}, B_{1}^{T}, \ldots, B_{1}^{T}}_{N}]^{T}, \quad \tilde{B}_{2}=[\underbrace{B_{2}^{T}, B_{2}^{T}, \ldots, B_{2}^{T}}_{N}]^{T} \\
& \tilde{D}=[\underbrace{D^{T}, D^{T}, \ldots, D^{T}}_{N}]^{T}, \tilde{M}_{0}=[\underbrace{M_{0}^{T}, M_{0}^{T}, \ldots, M_{0}^{T}}_{N}]^{T}
\end{aligned}
$$




$$
\begin{gathered}
\bar{C}=\left[\begin{array}{c}
C_{1} \\
C_{2} \\
\vdots \\
C_{N}
\end{array}\right] \quad F(k, \tilde{x}(k))=\left[\begin{array}{c}
\tilde{f}\left(k, \tilde{x}_{1}(k)\right) \\
\tilde{f}\left(k, \tilde{x}_{2}(k)\right) \\
\vdots \\
\tilde{f}\left(k, \tilde{x}_{N}(k)\right)
\end{array}\right] \\
\tilde{M}=\left[\begin{array}{c}
M_{1} \\
M_{2} \\
\vdots \\
M_{N}
\end{array}\right] \quad G(k, \tilde{x}(k))=\left[\begin{array}{c}
\tilde{g}\left(k, \tilde{x}_{1}(k)\right) \\
\tilde{g}\left(k, \tilde{x}_{2}(k)\right) \\
\vdots \\
\tilde{g}\left(k, \tilde{x}_{N}(k)\right)
\end{array}\right]
\end{gathered}
$$

and $\bar{K}=\left(l_{i j} K_{i j}\right)_{N \times N}$ is a sparse matrix satisfying $\bar{K} \in \mathscr{W}_{n \times m}$, where $\mathscr{W}_{n \times m}$ is defined as

$$
\begin{array}{r}
\mathscr{W}_{n \times m}=\left\{\bar{U}=\left[U_{i j}\right] \in \mathbb{R}^{n N \times m N} \mid U_{i j} \in \mathbb{R}^{n \times m},\right. \\
\left.U_{i j}=0 \text { if } j \notin \mathcal{N}_{i}\right\} .
\end{array}
$$

In terms of Assumption 2, one can easily obtain

$$
\|F(k, \tilde{x}(k))\| \leq\left\|\tilde{\Sigma}_{1} \tilde{x}(k)\right\|, \quad \| G\left(t, \tilde{x}(k)\|\leq\| \tilde{\Sigma}_{2} \tilde{x}(k) \|\right.
$$

where $\tilde{\Sigma}_{1}=\operatorname{diag}\left(\Sigma_{1}, \Sigma_{1}, \ldots, \Sigma_{1}\right)$ and $\tilde{\Sigma}_{2}=\operatorname{diag}\left(\Sigma_{2}, \Sigma_{2}\right.$, $\left.\ldots, \Sigma_{2}\right)$.

For notational simplicity, in the rest of this paper, let $\mathbf{e}_{i}=$ $\operatorname{col}\left(0_{m}, \ldots, I_{m}, \ldots, 0_{m}\right) \in \mathbb{R}^{m N \times m}$ be the matrix column with the $i$ th block as the identity matrix $I_{m}$ and the others as $0_{m}$. Also, denote

$$
\hat{K}=\operatorname{diag}\left(\bar{K}_{1}, \bar{K}_{2}, \ldots, \bar{K}_{N}\right)
$$

where $\bar{K}_{i}=\operatorname{col}\left(l_{1 i} K_{1 i}, l_{2 i} K_{2 i}, \ldots, l_{N i} K_{N i}\right)$. Obviously, for $i=1,2, \ldots, N$, the equalities $\bar{K}_{i}=\bar{K} \mathbf{e}_{i}$ hold.

By letting

$$
\eta(k)=\left[\begin{array}{c}
x(k) \\
\tilde{x}(k)
\end{array}\right]
$$

we get the following augmented system with the combination of (1) and (8):

$$
\begin{aligned}
\eta(k+1)= & \mathcal{Y}(k)+(\delta(k)-\bar{\delta}) \mathcal{B}_{2} \mathscr{F}(k, \eta(k)) \\
& +\mathcal{M} \eta(k) \omega_{0}(k)-\mathcal{C}(k) \eta(k)
\end{aligned}
$$

where $\mathcal{Y}(k)=\mathcal{A} \eta(k)+\mathcal{D} \eta(k-\tau(k))+\mathcal{B}_{1} \mathscr{F}(k, \eta(k))$ and $\mathcal{A}=\operatorname{diag}\left(A, I_{N} \otimes A-\bar{K} \Gamma \tilde{C}\right)$

$$
\begin{aligned}
& \mathcal{D}=\left[\begin{array}{cc}
D & 0 \\
\tilde{D} & 0
\end{array}\right] \quad \mathcal{B}_{2}=\left[\begin{array}{llll}
B_{1} & -B_{2} & 0 & 0 \\
\tilde{B}_{1} & -\tilde{B}_{2} & 0 & 0
\end{array}\right] \\
& \mathcal{B}_{1}=\left[\begin{array}{cccc}
\bar{\delta} B_{1} & (1-\bar{\delta}) B_{2} & 0 & 0 \\
0 & 0 & \bar{\delta}\left(I_{N} \otimes B_{1}\right) & \mathbf{B}_{2}
\end{array}\right] \\
& \mathcal{M}=\left[\begin{array}{cc}
M_{0} & 0 \\
\tilde{M}_{0} & 0
\end{array}\right] \quad \mathscr{F}(k, \eta(k))=\left[\begin{array}{c}
f(k, x(k)) \\
g(k, x(k)) \\
F(k, \tilde{x}(x)) \\
G(k, \tilde{x}(k))
\end{array}\right] \\
& \mathcal{C}(k)=\left[\begin{array}{cc}
0 & 0 \\
\bar{K}(\Gamma(k)-\Gamma) \bar{C}+\bar{K} \bar{\omega}(k) \tilde{M} & 0
\end{array}\right]
\end{aligned}
$$

and $\mathbf{B}_{2}=(1-\bar{\delta})\left(I_{N} \otimes B_{2}\right)$.

By Assumptions 1, 2, and (10), one can easily conclude that the following inequality holds:

$$
\|\mathscr{F}(k, \eta(k))\| \leq\|\widehat{\Sigma} \eta(k)\|
$$

where

$$
\widehat{\Sigma}=\left[\begin{array}{cc}
\Sigma_{1} & 0 \\
\Sigma_{2} & 0 \\
0 & \tilde{\Sigma}_{1} \\
0 & \tilde{\Sigma}_{2}
\end{array}\right]
$$

and $\tilde{\Sigma}_{1}, \tilde{\Sigma}_{2}$ are as defined in (10).

The initial condition associated with (12) is given as

$$
\eta(s)=\varphi(s) \in \mathbb{R}^{n(N+1)} \quad s=-\tau_{2},-\tau_{2}+1, \ldots, 0
$$

where $\varphi(\cdot)$ is independent of the processes $\delta(\cdot), \gamma_{i}(\cdot)(i=$ $1,2, \ldots, N)$ and $\omega_{j}(\cdot)(j=0,1, \ldots, N)$.

Definition 1: The system (6) is said to be a convergent distributed state estimator of the networked dynamic system (1) with measurement outputs (2) if the estimation error system (12) is globally asymptotically stable in the mean square sense, i.e., for any initial condition $\varphi(\cdot)$, the corresponding solution $\{\eta(k) ; k \in \mathbb{N}\}$ satisfies

$$
\lim _{k \rightarrow \infty} \mathbb{E}\left\{\|\eta(k)\|^{2}\right\}=0 .
$$

\section{MAIN RESUlTS}

In this paper, we are aiming at establishing sufficient criteria for the discrete-time nonlinear system (12) with stochastic disturbances to be globally asymptotically stable in the mean square sense. By resorting to the Lyapunov functional stability theory and the stochastic analysis technique, verifiable conditions are derived step by step.

Theorem 1: Under Assumptions 1 and 2, for the given $\bar{K}$, the network in (12) with time-varying delay is globally asymptotically stable in the mean square sense if there exist matrices $P_{j}>0(j=1,2, \ldots, N+1), \mathcal{Q}>0$, matrices $\mathcal{W}$, $\mathcal{R}$, and scalar $\varepsilon>0$ such that

$$
\mathcal{P} \triangleq\left[\begin{array}{cc}
P_{1} & \mathcal{R} \\
* & \mathcal{P}_{2}
\end{array}\right]>0 \text { and } \Xi+\operatorname{Sym}(\mathcal{W} \mathcal{T})<0
$$

where $\mathcal{P}_{2}=\operatorname{diag}\left(P_{2}, P_{3}, \ldots, P_{N+1}\right)$

$$
\begin{aligned}
\mathcal{T}= & {\left[\begin{array}{cccc}
-\mathcal{A} & -\mathcal{D} & -\mathcal{B}_{1} & I_{n(N+1)}
\end{array}\right] } \\
\Xi= & {\left[\begin{array}{cccc}
\Xi_{11} & \mathcal{A}^{T} \mathcal{P} \mathcal{D} & \mathcal{A}^{T} \mathcal{P} \mathcal{B}_{1} & 0 \\
* & -\mathcal{Q}^{T} \mathcal{D}^{T} \mathcal{P} \mathcal{D} & \mathcal{D}^{T} \mathcal{P} \mathcal{B}_{1} & 0 \\
* & * & \Xi_{33} & 0 \\
* & * & * & 0
\end{array}\right] } \\
\Xi_{11}= & \left(\tau_{2}-\tau_{1}+1\right) \mathcal{Q}+\varepsilon \widehat{\Sigma}^{T} \widehat{\Sigma}+\mathcal{A}^{T} \mathcal{P} \mathcal{A}-\mathcal{P} \\
& +\mathcal{M}^{T} \mathcal{P} \mathcal{M}+\sum_{i=1}^{N}\left(\mathcal{C}_{1 i}^{T} \mathcal{P} \mathcal{C}_{1 i}+\mathcal{C}_{2 i}^{T} \mathcal{P} \mathcal{C}_{2 i}\right) \\
\Xi_{33}= & -\varepsilon I_{2 n(N+1)}+\mathcal{B}_{1}^{T} \mathcal{P} \mathcal{B}_{1}+\bar{\delta}(1-\bar{\delta}) \mathcal{B}_{2}^{T} \mathcal{P} \mathcal{B}_{2}
\end{aligned}
$$

and

$\mathcal{C}_{1 i}=\left[\begin{array}{cc}0 & 0 \\ \sqrt{\bar{\gamma}_{i}\left(1-\bar{\gamma}_{i}\right)} \bar{K} \mathbf{e}_{i} C_{i} & 0\end{array}\right] \quad \mathcal{C}_{2 i}=\left[\begin{array}{cc}0 & 0 \\ \bar{K} \mathbf{e}_{i} M_{i} & 0\end{array}\right]$

Proof: $\quad$ Let $\mathscr{X}(k) \triangleq\{\eta(k), \eta(k-1), \ldots, \eta(k-$ $\left.\tau(k)), \ldots, \eta\left(k-\tau_{2}\right)\right\}$, and consider the following Lyapunov 
functional candidate for the augmented estimation error system (12):

$$
\begin{aligned}
V(\mathscr{X}(k))= & V_{1}(\mathscr{X}(k))+V_{2}(\mathscr{X}(k))+V_{3}(\mathscr{X}(k)) \\
= & \eta^{T}(k) \mathcal{P} \eta(k)+\sum_{i=k-\tau(k)}^{k-1} \eta^{T}(i) \mathcal{Q} \eta(i) \\
& +\sum_{j=1-\tau_{2}}^{-\tau_{1}} \sum_{i=k+j}^{k-1} \eta^{T}(i) \mathcal{Q} \eta(i)
\end{aligned}
$$

where $\mathcal{P}>0, \mathcal{Q}>0$ are matrices to be determined and $\mathcal{P}$ is just as defined in (15).

Along the solutions of system (12), calculating the difference of $V_{j}(\mathscr{X}(k))(j=1,2,3)$ and taking the mathematical expectation conditional on $\mathscr{X}(k)$, we have

$$
\begin{aligned}
& \mathbb{E}\left\{\Delta V_{1}(\mathscr{X}(k)) \mid \mathscr{X}(k)\right\} \\
= & \mathbb{E}\left\{\left(V_{1}(\mathscr{X}(k+1))-V_{1}(\mathscr{X}(k))\right) \mid \mathscr{X}(k)\right\} \\
= & \mathbb{E}\left\{V_{1}(\mathscr{X}(k+1)) \mid \mathscr{X}(k)\right\}-V_{1}(\mathscr{X}(k)) \\
= & \mathbb{E}\left\{\left(\eta^{T}(k)\left(\mathcal{A}^{T} \mathcal{P} \mathcal{A}-\mathcal{P}\right) \eta(k)+\eta^{T}(k-\tau(k)) \mathcal{D}^{T} \mathcal{P}\right.\right. \\
& \times \mathcal{D} \eta(k-\tau(k))+\mathscr{F}^{T}(k, \eta(k)) \mathcal{B}_{1}^{T} \mathcal{P} \mathcal{B}_{1} \mathscr{F}(k, \eta(k)) \\
& +2 \eta^{T}(k) \mathcal{A}^{T} \mathcal{P}\left[\mathcal{D} \eta(k-\tau(k))+\mathcal{B}_{1} \mathscr{F}(k, \eta(k))\right] \\
& +2 \eta^{T}(k-\tau(k)) \mathcal{D}^{T} \mathcal{P} \mathcal{B}_{1} \mathscr{F}(k, \eta(k)) \\
& +2\left[\mathcal{A} \eta(k)+\mathcal{D} \eta(k-\tau(k))+\mathcal{B}_{1} \mathscr{F}(k, \eta(k))\right]^{T} \mathcal{P} \\
& \times\left[(\delta(k)-\bar{\delta}) \mathcal{B}_{2} \mathscr{F}(k, \eta(k))+\mathcal{M} \eta(k) \omega_{0}(k)-\mathcal{C}(k) \eta(k)\right] \\
& +2(\delta(k)-\bar{\delta}) \mathscr{F}^{T}(k, \eta(k)) \mathcal{B}_{2}^{T} \mathcal{P} \\
& \times\left[\mathcal{M} \eta(k) \omega_{0}(k)-\mathcal{C}(k) \eta(k)\right] \\
& -2 \omega_{0}(k) \eta^{T}(k) \mathcal{M}^{T} \mathcal{P C}(k) \eta(k) \\
& +\omega_{0}^{2}(k) \eta^{T}(k) \mathcal{M}^{T} \mathcal{P} \mathcal{M} \eta(k)+\eta^{T}(k) \mathcal{C}^{T}(k) \mathcal{P C}(k) \eta(k) \\
& \left.\left.+(\delta(k)-\bar{\delta})^{2} \mathscr{F}^{T}(k, \eta(k)) \mathcal{B}_{2}^{T} \mathcal{P} \mathcal{B}_{2} \mathscr{F}(k, \eta(k))\right) \mid \mathscr{X}(k)\right\} \\
= & \mathbb{E}\left\{\left(\eta^{T}(k)\left(\mathcal{A}^{T} \mathcal{P} \mathcal{A}-\mathcal{P}\right) \eta(k)+\eta^{T}(k-\tau(k)) \mathcal{D}^{T} \mathcal{P}\right.\right. \\
& \times \mathcal{D} \eta(k-\tau(k))+\mathscr{F}^{T}(k, \eta(k)) \mathcal{B}_{1}^{T} \mathcal{P} \mathcal{B}_{1} \mathscr{F}(k, \eta(k)) \\
& +2 \eta^{T}(k) \mathcal{A}^{T} \mathcal{P}\left[\mathcal{D} \eta(k-\tau(k))+\mathcal{B}_{1} \mathscr{F}(k, \eta(k))\right] \\
& +2 \eta^{T}(k-\tau(k)) \mathcal{D}^{T} \mathcal{P} \mathcal{B}_{1} \mathscr{F}(k, \eta(k)) \\
& +\bar{\delta}(1-\bar{\delta}) \mathscr{F}^{T}(k, \eta(k)) \mathcal{B}_{2}^{T} \mathcal{P} \mathcal{B}_{2} \mathscr{F}(k, \eta(k))+\eta^{T}(k) \mathcal{M}^{T} \\
& \left.\left.\times \mathcal{P} \mathcal{M} \eta(k)+\eta^{T}(k) \mathcal{C}^{T}(k) \mathcal{P C}(k) \eta(k)\right) \mid \mathscr{X}(k)\right\} \\
& \left.\mathbb{A} V_{2}(\mathscr{T}(k)) \mid \mathscr{X}(k)\right\}
\end{aligned}
$$$$
\mathbb{E}\left\{\Delta V_{2}(\mathscr{X}(k)) \mid \mathscr{X}(k)\right\}
$$$$
=\mathbb{E}\left\{\left(\sum_{i=k+1-\tau(k+1)}^{k}-\sum_{i=k-\tau(k)}^{k-1}\right) \eta^{T}(i) \mathcal{Q} \eta(i) \mid \mathscr{X}(k)\right\}
$$$$
=\mathbb{E}\left\{\left(\sum_{i=k+1-\tau(k+1)}^{k-\tau_{1}}+\sum_{i=k+1-\tau_{1}}^{k}-\sum_{i=k-\tau(k)}^{k-1}\right) \eta^{T}(i) \mathcal{Q} \eta(i) \mid \mathscr{X}(k)\right\}
$$$$
\leq \mathbb{E}\left\{\left(\sum_{i=k+1-\tau(k+1)}^{k-\tau_{1}} \eta^{T}(i) \mathcal{Q} \eta(i)+\eta^{T}(k) \mathcal{Q} \eta(k)\right.\right.
$$$$
\left.\left.-\eta^{T}(k-\tau(k)) \mathcal{Q} \eta(k-\tau(k))\right) \mid \mathscr{X}(k)\right\}
$$$$
\leq \mathbb{E}\left\{\left(\sum_{i=k+1-\tau_{2}}^{k-\tau_{1}} \eta^{T}(i) \mathcal{Q} \eta(i)+\eta^{T}(k) \mathcal{Q} \eta(k)\right.\right.
$$

$$
\begin{aligned}
& \left.\left.-\eta^{T}(k-\tau(k)) \mathcal{Q} \eta(k-\tau(k))\right) \mid \mathscr{X}(k)\right\} \\
& \mathbb{E}\left\{\Delta V_{3}(\mathscr{X}(k)) \mid \mathscr{X}(k)\right\} \\
= & \mathbb{E}\left\{\sum_{j=1-\tau_{2}}^{-\tau_{1}}\left(\sum_{i=k+1+j}^{k}-\sum_{i=k+j}^{k-1}\right) \eta^{T}(i) \mathcal{Q} \eta(i) \mid \mathscr{X}(k)\right\} \\
= & \mathbb{E}\left\{\sum_{j=1-\tau_{2}}^{-\tau_{1}}\left(\eta^{T}(k) \mathcal{Q} \eta(k)-\eta^{T}(k+j) \mathcal{Q} \eta(k+j)\right) \mid \mathscr{X}(k)\right\} \\
= & \mathbb{E}\left\{\left(\left(\tau_{2}-\tau_{1}\right) \eta^{T}(k) \mathcal{Q} \eta(k)-\sum_{i=k+1-\tau_{2}}^{k-\tau_{1}} \eta^{T}(i) \mathcal{Q} \eta(i)\right) \mid \mathscr{X}(k)\right\} .
\end{aligned}
$$

From the definition of matrix $\mathcal{C}(k)$ defined in (12) and the mutually independent properties of processes $\gamma_{j}(k)$ and $\omega_{j}(k)(j=1,2, \ldots, N)$, we can derive

$$
\begin{aligned}
& \mathbb{E}\left\{\eta^{T}(k) \mathcal{C}^{T}(k) \mathcal{P C}(k) \eta(k) \mid \mathscr{X}(k)\right\} \\
= & \mathbb{E}\left\{x^{T}(x)(\bar{K}(\Gamma(k)-\Gamma) \bar{C}+\bar{K} \bar{\omega}(k) \tilde{M})^{T} \mathcal{P}_{2}\right. \\
& \times(\bar{K}(\Gamma(k)-\Gamma) \bar{C}+\bar{K} \bar{\omega}(k) \tilde{M}) x(k) \mid \mathscr{X}(k)\} \\
= & \mathbb{E}\left\{\left(x^{T}(k) \tilde{M}^{T} \bar{\omega}^{T}(k) \bar{K}^{T} \mathcal{P}_{2} \bar{K} \bar{\omega}(k) \tilde{M} x(k)\right.\right. \\
& +x^{T}(k) \bar{C}^{T}(\Gamma(k)-\Gamma)^{T} \bar{K}^{T} \mathcal{P}_{2} \bar{K}(\Gamma(k)-\Gamma) \bar{C} x(k) \\
& +2 x^{T}(k) \tilde{M}^{T} \bar{\omega}^{T}(k) \bar{K}^{T} \mathcal{P}_{2} \\
& \times \bar{K}(\Gamma(k)-\Gamma) \bar{C} x(k)) \mid \mathscr{X}(k)\} \\
= & \mathbb{E}\left\{\left(x^{T}(k) \tilde{M}^{T} \bar{\omega}^{T}(k) \bar{K}^{T} \mathcal{P}_{2} \bar{K} \bar{\omega}(k) \tilde{M} x(k)\right.\right. \\
& +x^{T}(k) \bar{C}^{T}(\Gamma(k)-\Gamma)^{T} \bar{K}^{T} \mathcal{P}_{2} \\
& \times \bar{K}(\Gamma(k)-\Gamma) \bar{C} x(k)) \mid \mathscr{X}(k)\}
\end{aligned}
$$

in which

$$
\begin{aligned}
& \mathbb{E}\left\{x^{T}(k) \tilde{M}^{T} \bar{\omega}^{T}(k) \bar{K}^{T} \mathcal{P}_{2} \bar{K} \bar{\omega}(k) \tilde{M} x(k) \mid \mathscr{X}(k)\right\} \\
= & \mathbb{E}\left\{x ^ { T } ( k ) \left(\sum_{i=1}^{N}\left(\sum_{j \in \mathcal{N}_{i}} l_{i j} K_{i j} M_{j} \omega_{j}(k)\right)^{T} P_{i+1}\right.\right. \\
& \left.\left.\times\left(\sum_{j \in \mathcal{N}_{i}} l_{i j} K_{i j} M_{j} \omega_{j}(k)\right)\right) x(k) \mid \mathscr{X}(k)\right\} \\
= & \mathbb{E}\left\{x ^ { T } ( k ) \left(\sum_{i=1}^{N} \sum_{j \in \mathcal{N}_{i}} l_{i j}^{2}\left(K_{i j} M_{j}\right)^{T} P_{i+1}\right.\right. \\
& \left.\left.\times\left(K_{i j} M_{j}\right)\right) x(k) \mid \mathscr{X}(k)\right\} \\
= & \mathbb{E}\left\{x^{T}(k)\left(\sum_{i=1}^{N} M_{i}^{T} \bar{K}_{i}^{T} \mathcal{P}_{2} \bar{K}_{i} M_{i}\right) x(k) \mid \mathscr{X}(k)\right\} \\
= & \mathbb{E}\left\{x^{T}(k) \tilde{M}^{T} \hat{K}^{T}\left(I_{N} \otimes \mathcal{P}_{2}\right) \hat{K} \tilde{M} x(k) \mid \mathscr{X}(k)\right\} \\
= & \mathbb{E}\left\{\sum_{i=1}^{N} \eta^{T}(k) \mathcal{C}_{2 i}^{T} \mathcal{P} \mathcal{C}_{2 i} \eta(k) \mid \mathscr{X}(k)\right\} .
\end{aligned}
$$

Similarly, the following equality holds:

$$
\begin{aligned}
& \mathbb{E}\left\{x^{T}(k) \bar{C}^{T}(\Gamma(k)-\Gamma)^{T} \bar{K}^{T} \mathcal{P}_{2}\right. \\
& \times \bar{K}(\Gamma(k)-\Gamma) \bar{C} x(k) \mid \mathscr{X}(k)\}
\end{aligned}
$$




$$
\begin{aligned}
& =\mathbb{E}\left\{x^{T}(k) \bar{C}^{T} \tilde{\Gamma}^{T} \hat{K}^{T}\left(I_{N} \otimes \mathcal{P}_{2}\right) \hat{K} \tilde{\Gamma} \bar{C} x(k) \mid \mathscr{X}(k)\right\} \\
& =\mathbb{E}\left\{\sum_{i=1}^{N} \eta^{T}(k) \mathcal{C}_{1 i}^{T} \mathcal{P} \mathcal{C}_{1 i} \eta(k) \mid \mathscr{X}(k)\right\}
\end{aligned}
$$

where $\tilde{\Gamma}=\operatorname{diag}\left(\sqrt{\bar{\gamma}_{1}\left(1-\bar{\gamma}_{1}\right)} I_{m}, \sqrt{\bar{\gamma}_{2}\left(1-\bar{\gamma}_{2}\right)} I_{m}, \ldots\right.$, $\left.\sqrt{\bar{\gamma}_{N}\left(1-\bar{\gamma}_{N}\right)} I_{m}\right)$.

Substituting (21) and (22) into (20), we have

$$
\begin{aligned}
& \mathbb{E}\left\{\eta^{T}(k) \mathcal{C}^{T}(k) \mathcal{P C}(k) \eta(k) \mid \mathscr{X}(k)\right\} \\
= & \mathbb{E}\left\{\eta^{T}(k) \sum_{i=1}^{N}\left(\mathcal{C}_{2 i}^{T} \mathcal{P} \mathcal{C}_{2 i}+\mathcal{C}_{1 i}^{T} \mathcal{P C} \mathcal{C}_{1 i}\right) \eta(k) \mid \mathscr{X}(k)\right\} .
\end{aligned}
$$

On the other hand, based on (12), for any matrix $\mathcal{W}$ of appropriate dimensions, one has

$$
\begin{aligned}
& 2 \xi^{T}(k) \mathcal{W}[\mathcal{Y}(k)-\mathcal{A} \eta(k) \\
&\left.-\mathcal{D} \eta(k-\tau(k))-\mathcal{B}_{1} \mathscr{F}(k, \eta(k))\right]=0
\end{aligned}
$$

where $\xi^{T}(k)=\left[\eta^{T}(k), \eta^{T}(k-\tau(k)), \mathscr{F}^{T}(k, \eta(k)), \mathcal{Y}^{T}(k)\right]$. Furthermore, (13) ensures that for any positive scalar $\varepsilon$, the following inequality holds:

$$
\varepsilon \eta^{T}(k) \widehat{\Sigma}^{T} \widehat{\Sigma} \eta(k)-\varepsilon \mathscr{F}^{T}(k, \eta(k)) \mathscr{F}(k, \eta(k)) \geq 0 .
$$

Therefore, adding the left side of (24) to (17), and by considering (18), (19), (23), and (25), we eventually obtain

$$
\mathbb{E}\{\Delta V(\mathscr{X}(k)) \mid \mathscr{X}(k)\} \leq \xi^{T}(k)(\Xi+\operatorname{Sym}(\mathcal{W T})) \xi(k)
$$

where $\Xi$ is defined in $(15)$ and $\mathcal{T}=\left[-\mathcal{A},-\mathcal{D},-\mathcal{B}_{1}, I_{n(N+1)}\right]$. The remaining part of the proof is similar to those in [37] and [30] and so omitted here for simplicity, and the proof is then completed.

Remark 3: To reflect a more realistic situation in practice, different Brownian motions $\omega_{0}(\cdot)$ and $\omega_{i}(\cdot)(i=1,2, \ldots, N)$ are introduced, respectively, for describing the dynamics of the target system and the sensor network. This brings some difficulty in dealing with the stochastic disturbances reflected by the term $\mathcal{C}(k) \eta(k)$ in (12). In the proof of Theorem 1, we solve this problem by rearranging the block matrices in $\bar{K}$ and elegantly choosing $\mathcal{P}_{2}$ with special structure.

After conducting the dynamic analysis in Theorem 1 for the augmented estimation error system (12), we are in a position to deal with the problem of designing the distributed state estimator (6) for the networked target system (1) with sensor network (2). Before processing on, the following lemma will be utilized in establishing our criteria.

Lemma 1: Let $\mathbf{S}=\operatorname{diag}\left(S_{11}, S_{22}, \ldots, S_{N N}\right)$, with $S_{i i} \in$ $\mathbb{R}^{n \times n}(i=1,2, \ldots, N)$ being invertible matrices. If $X=\mathbf{S} \bar{U}$ for $\bar{U} \in \mathbb{R}^{n N \times m N}$, then we have $\bar{U} \in \mathscr{W}_{n \times m} \Leftrightarrow X \in \mathscr{W}_{n \times m}$.

Theorem 2: Under Assumptions 1 and 2, the system (6) is a convergent distributed state estimator of the networked dynamic system (1) with measurement outputs (2) if there exist matrices $P_{j}>0(j=1,2, \ldots, N+1), Q_{11}>0, \mathcal{Q}_{22}>0$, matrices $Q_{12}, W_{i 11}, W_{i 21}(i=1,4), \mathcal{X} \in \mathscr{W}_{n \times m}$, and scalar $\varepsilon>0$ such that

$\mathcal{Q} \triangleq\left[\begin{array}{cc}Q_{11} & Q_{12} \\ * & \mathcal{Q}_{22}\end{array}\right]>0, \Phi+\Theta\left(I_{2 N+1} \otimes \mathcal{P}_{2}^{-1}\right) \Theta^{T}<0$ where $\mathcal{P}_{2}=\operatorname{diag}\left(P_{2}, P_{3}, \ldots, P_{N+1}\right)$

$$
\begin{aligned}
& \Theta=\left[\begin{array}{ccc}
0 & \aleph_{1}^{T} & \aleph_{2}^{T} \\
\left(I_{N} \otimes A\right)^{T} \mathcal{P}_{2}-\tilde{C}^{T} \Gamma^{T} \mathcal{X}^{T} & 0 & 0 \\
0 & 0 & 0
\end{array}\right] \\
& \aleph_{1}=\left[\begin{array}{c}
\mathcal{X} \mathbf{e}_{1} M_{1} \\
\mathcal{X} \mathbf{e}_{2} M_{2} \\
\vdots \\
\mathcal{X} \mathbf{e}_{N} M_{N}
\end{array}\right] \quad \aleph_{2}=\left[\begin{array}{c}
\sqrt{\bar{\gamma}_{1}\left(1-\bar{\gamma}_{1}\right)} \mathcal{X} \mathbf{e}_{1} C_{1} \\
\sqrt{\bar{\gamma}_{2}\left(1-\bar{\gamma}_{2}\right)} \mathcal{X} \mathbf{e}_{2} C_{2} \\
\vdots \\
\sqrt{\bar{\gamma}_{N}\left(1-\bar{\gamma}_{N}\right)} \mathcal{X} \mathbf{e}_{N} C_{N}
\end{array}\right] \\
& \Phi=\left[\begin{array}{cccccc}
\Phi_{11} & \Phi_{12} & \Phi_{13} & 0 & \Phi_{15} & \Phi_{16} \\
* & \Phi_{22} & \Phi_{23} & 0 & -\bar{\delta} W_{121} B_{1} & \Phi_{26} \\
* & * & \Phi_{33} & -Q_{12} & \bar{\delta} D^{T} P_{1} B_{1} & \Phi_{36} \\
* & * & * & -\mathcal{Q}_{22} & 0 & 0 \\
* & * & * & * & \Phi_{55} & \Phi_{56} \\
* & * & * & * & * & \Phi_{66} \\
* & * & * & * & * & * \\
* & * & * & * & * & * \\
* & * & * & * & * & * \\
* & * & * & * & * & * \\
0 & 0 & W_{111}-A^{T} W_{411}^{T} & -A^{T} W_{421}^{T} \\
\Phi_{27} & \Phi_{28} & W_{121} & \Phi_{2,10} \\
\Phi_{37} & \Phi_{38} & -D^{T} W_{411}^{T} & \Phi_{3,10} \\
0 & 0 & 0 & 0 \\
0 & 0 & -\bar{\delta} B_{1}^{T} W_{411}^{T} & -\bar{\delta} B_{1}^{T} W_{421}^{T} \\
0 & 0 & -(1-\bar{\delta}) B_{2}^{T} W_{411}^{T} & \Phi_{6,10} \\
\Phi_{77} & \Phi_{78} & 0 & \Phi_{7,10} \\
* & \Phi_{88} & 0 & \Phi_{8,10} \\
* & * & \operatorname{Sym}\left(W_{411}\right) & W_{421}^{T} \\
* & * & * & -2 \mathcal{P}_{2}
\end{array}\right]
\end{aligned}
$$

and

$$
\begin{aligned}
\Phi_{11}= & A^{T} P_{1} A-P_{1}+M_{0}^{T} P_{1} M_{0}+\tilde{M}_{0}^{T} \mathcal{P}_{2} \tilde{M}_{0} \\
& +\varepsilon \Sigma_{1}^{T} \Sigma_{1}+\left(\tau_{2}-\tau_{1}+1\right) Q_{11}+\varepsilon \Sigma_{2}^{T} \Sigma_{2} \\
& -\operatorname{Sym}\left(W_{111} A\right) \\
\Phi_{12}= & \left(\tau_{2}-\tau_{1}+1\right) Q_{12}-A^{T} W_{121}^{T} \\
\Phi_{13}= & A^{T} P_{1} D-W_{111} D, \quad \Phi_{15}=\bar{\delta}\left(A^{T} P_{1}-W_{111}\right) B_{1} \\
\Phi_{16}= & (1-\bar{\delta})\left(A^{T} P_{1}-W_{111}\right) B_{2} \\
\Phi_{22}= & -\mathcal{P}_{2}+\left(\tau_{2}-\tau_{1}+1\right) \mathcal{Q}_{22}+\varepsilon \tilde{\Sigma}_{1}^{T} \tilde{\Sigma}_{1}+\varepsilon \tilde{\Sigma}_{2}^{T} \tilde{\Sigma}_{2} \\
& -\operatorname{Sym}\left(\mathcal{P}_{2}\left(I_{N} \otimes A\right)-\mathcal{X} \Gamma \tilde{C}\right) \\
\Phi_{23}= & \left(I_{N} \otimes A\right)^{T} \mathcal{P}_{2} \tilde{D}-\tilde{C}^{T} \Gamma^{T} \mathcal{X}^{T} \tilde{D}-W_{121} D-\mathcal{P}_{2} \tilde{D} \\
\Phi_{26}= & -(1-\bar{\delta}) W_{121} B_{2} \\
\Phi_{27}= & \bar{\delta}\left(I_{N} \otimes A\right)^{T} \mathcal{P}_{2}\left(I_{N} \otimes B_{1}\right)-\bar{\delta} \mathcal{P}_{2}\left(I_{N} \otimes B_{1}\right) \\
& -\bar{\delta} \tilde{C}^{T} \Gamma^{T} \mathcal{X}^{T}\left(I_{N} \otimes B_{1}\right) \\
\Phi_{28}= & (1-\bar{\delta})\left(\left(I_{N} \otimes A\right)^{T} \mathcal{P}_{2}\left(I_{N} \otimes B_{2}\right)\right. \\
& \left.-\tilde{C}^{T} \Gamma^{T} \mathcal{X}^{T}\left(I_{N} \otimes B_{2}\right)-\mathcal{P}_{2}\left(I_{N} \otimes B_{2}\right)\right) \\
\Phi_{2,10}= & \mathcal{P}_{2}+\left(I_{N} \otimes A\right)^{T} \mathcal{P}_{2}-\tilde{C}^{T} \Gamma^{T} \mathcal{X}^{T} \\
\Phi_{33}= & D^{T} P_{1} D-Q_{11}+\tilde{D}^{T} \mathcal{P}_{2} \tilde{D} \\
\Phi_{36}= & (1-\bar{\delta}) D^{T} P_{1} B_{2}, \quad \Phi_{37}=\bar{\delta} \tilde{D}^{T} \mathcal{P}_{2}\left(I_{N} \otimes B_{1}\right) \\
\Phi_{38}= & (1-\bar{\delta}) \tilde{D}^{T} \mathcal{P}_{2}\left(I_{N} \otimes B_{2}\right) \\
\Phi_{3,10}= & \tilde{D}^{T} \mathcal{P}_{2}-D^{T} W_{421}^{T}
\end{aligned}
$$




$$
\begin{aligned}
\Phi_{55} & =\bar{\delta} B_{1}^{T} P_{1} B_{1}-\varepsilon I+\bar{\delta}(1-\bar{\delta}) \tilde{B}_{1}^{T} \mathcal{P}_{2} \tilde{B}_{1} \\
\Phi_{56} & =-\bar{\delta}(1-\bar{\delta}) \tilde{B}_{1}^{T} \mathcal{P}_{2} \tilde{B}_{2}, \quad \Phi_{6,10}=-(1-\bar{\delta}) B_{2}^{T} W_{421}^{T} \\
\Phi_{66} & =(1-\bar{\delta}) B_{2}^{T} P_{1} B_{2}+\bar{\delta}(1-\bar{\delta}) \tilde{B}_{2}^{T} \mathcal{P}_{2} \tilde{B}_{2}-\varepsilon I \\
\Phi_{77} & =\bar{\delta}^{2}\left(I_{N} \otimes B_{1}\right)^{T} \mathcal{P}_{2}\left(I_{N} \otimes B_{1}\right)-\varepsilon I \\
\Phi_{78} & =\bar{\delta}(1-\bar{\delta})\left(I_{N} \otimes B_{1}\right)^{T} \mathcal{P}_{2}\left(I_{N} \otimes B_{2}\right) \\
\Phi_{7,10} & =\bar{\delta}\left(I_{N} \otimes B_{1}\right)^{T} \mathcal{P}_{2} \\
\Phi_{88} & =(1-\bar{\delta})^{2}\left(I_{N} \otimes B_{2}\right)^{T} \mathcal{P}_{2}\left(I_{N} \otimes B_{2}\right)-\varepsilon I \\
\Phi_{8,10} & =(1-\bar{\delta})\left(I_{N} \otimes B_{2}\right)^{T} \mathcal{P}_{2}
\end{aligned}
$$

Moreover

$$
\bar{K}=\mathcal{P}_{2}^{-1} \mathcal{X}
$$

and, accordingly, the state estimator gains $K_{i j}(i=$ $1,2, \ldots, N, j \in \mathcal{N}_{i}$ ) can be derived from (9).

Proof: Let $\mathcal{P}=\operatorname{diag}\left(P_{1}, \mathcal{P}_{2}\right)$ and $\mathcal{P}_{2}=\operatorname{diag}\left(P_{2}\right.$, $\left.P_{3}, \ldots, P_{N+1}\right)$, obviously, $\mathcal{P}>0$. Furthermore, from Lemma 1, we know that $\bar{K}=\mathcal{P}_{2}^{-1} \mathcal{X} \in \mathscr{W}_{n \times m}$ under the condition that $\mathcal{X} \in \mathscr{W}_{n \times m}$.

From the definition of matrices $\mathcal{A}, \mathcal{D}, \mathcal{B}_{1}, \mathcal{B}_{2}, \mathcal{M}, \widehat{\Sigma}, \mathcal{C}_{1 i}$, and $\mathcal{C}_{2 i}(i=1,2, \ldots, N)$, and by computation, it is not difficult to obtain

$$
\begin{aligned}
& \mathcal{A}^{T} \mathcal{P} \mathcal{A}=\operatorname{diag}\left(A^{T} P_{1} A,\left(\mathcal{P}_{2}\left(I_{N} \otimes A\right)-\mathcal{X} \Gamma \tilde{C}\right)^{T} \mathcal{P}_{2}^{-1}\right. \\
& \left.\times\left(\mathcal{P}_{2}\left(I_{N} \otimes A\right)-\mathcal{X} \Gamma \tilde{C}\right)\right) \\
& \mathcal{D}^{T} \mathcal{P} \mathcal{D}=\operatorname{diag}\left(D^{T} P_{1} D+\tilde{D}^{T} \mathcal{P}_{2} \tilde{D}, 0\right) \\
& \mathcal{M}^{T} \mathcal{P} \mathcal{M}=\operatorname{diag}\left(M_{0}^{T} P_{1} M_{0}+\tilde{M}_{0}^{T} \mathcal{P}_{2} \tilde{M}_{0}, 0\right) \\
& \widehat{\Sigma}^{T} \widehat{\Sigma}=\operatorname{diag}\left(\Sigma_{1}^{T} \Sigma_{1}+\Sigma_{2}^{T} \Sigma_{2}, \tilde{\Sigma}_{1}^{T} \tilde{\Sigma}_{1}+\tilde{\Sigma}_{2}^{T} \tilde{\Sigma}_{2}\right) \\
& \mathcal{C}_{1 i}^{T} \mathcal{P} \mathcal{C}_{1 i}=\operatorname{diag}\left(\bar{\gamma}_{i}\left(1-\bar{\gamma}_{i}\right)\left(\mathcal{X} \mathbf{e}_{i} C_{i}\right)^{T} \mathcal{P}_{2}^{-1}\left(\mathcal{X} \mathbf{e}_{i} C_{i}\right), 0\right) \\
& \mathcal{C}_{2 i}^{T} \mathcal{P} \mathcal{C}_{2 i}=\operatorname{diag}\left(\left(\mathcal{X} \mathbf{e}_{i} M_{i}\right)^{T} \mathcal{P}_{2}^{-1}\left(\mathcal{X} \mathbf{e}_{i} M_{i}\right), 0\right) \\
& \mathcal{A}^{T} \mathcal{P} \mathcal{D}=\left[\begin{array}{cc}
A^{T} P_{1} D & 0 \\
\left(\mathcal{P}_{2}\left(I_{N} \otimes A\right)-\mathcal{X} \Gamma \tilde{C}\right)^{T} \tilde{D} & 0
\end{array}\right] \\
& \mathcal{A}^{T} \mathcal{P} \mathcal{B}_{1}=\left[\begin{array}{cccc}
\bar{\delta} A^{T} P_{1} B_{1} & (1-\bar{\delta}) A^{T} P_{1} B_{2} & 0 & 0 \\
0 & 0 & \Upsilon_{1} & \Upsilon_{2}
\end{array}\right] \\
& \mathcal{D}^{T} \mathcal{P} \mathcal{B}_{1}=\left[\begin{array}{cccc}
\bar{\delta} D^{T} P_{1} B_{1} & (1-\bar{\delta}) D^{T} P_{1} B_{2} & \Upsilon_{3} & \Upsilon_{4} \\
0 & 0 & 0 & 0
\end{array}\right] \\
& \mathcal{B}_{1}^{T} \mathcal{P} \mathcal{B}_{1}=\left[\begin{array}{cc}
\bar{\delta}^{2} B_{1}^{T} P_{1} B_{1} & \bar{\delta}(1-\bar{\delta}) B_{1}^{T} P_{1} B_{2} \\
* & (1-\bar{\delta})^{2} B_{2}^{T} P_{1} B_{2} \\
* & * \\
* & *
\end{array}\right. \\
& \begin{array}{ll}
0 & 0 \\
0 & 0
\end{array} \\
& \left.\begin{array}{cc}
\bar{\delta}^{2}\left(I_{N} \otimes B_{1}\right)^{T} \mathcal{P}_{2}\left(I_{N} \otimes B_{1}\right) & \Upsilon_{5} \\
* & \Upsilon_{6}
\end{array}\right] \\
& \mathcal{B}_{2}^{T} \mathcal{P} \mathcal{B}_{2}=\left[\begin{array}{cccc}
B_{1}^{T} P_{1} B_{1}+\tilde{B}_{1}^{T} \mathcal{P}_{2} \tilde{B}_{1} & \Upsilon_{7} & 0 & 0 \\
* & \Upsilon_{8} & 0 & 0 \\
* & * & 0 & 0 \\
* & * & * & 0
\end{array}\right]
\end{aligned}
$$

where

$$
\begin{aligned}
& \Upsilon_{1}=\bar{\delta}\left(\mathcal{P}_{2}\left(I_{N} \otimes A\right)-\mathcal{X} \Gamma \tilde{C}\right)^{T}\left(I_{N} \otimes B_{1}\right) \\
& \Upsilon_{2}=(1-\bar{\delta})\left(\mathcal{P}_{2}\left(I_{N} \otimes A\right)-\mathcal{X} \Gamma \tilde{C}\right)^{T}\left(I_{N} \otimes B_{2}\right) \\
& \Upsilon_{3}=\bar{\delta} \tilde{D}^{T} \mathcal{P}_{2}\left(I_{N} \otimes B_{1}\right) \\
& \Upsilon_{4}=(1-\bar{\delta}) \tilde{D}^{T} \mathcal{P}_{2}\left(I_{N} \otimes B_{2}\right)
\end{aligned}
$$

$$
\begin{aligned}
& \Upsilon_{5}=\bar{\delta}(1-\bar{\delta})\left(I_{N} \otimes B_{1}\right)^{T} \mathcal{P}_{2}\left(I_{N} \otimes B_{2}\right) ; \\
& \Upsilon_{6}=(1-\bar{\delta})^{2}\left(I_{N} \otimes B_{2}\right)^{T} \mathcal{P}_{2}\left(I_{N} \otimes B_{2}\right) ; \\
& \Upsilon_{7}=-B_{1}^{T} P_{1} B_{2}-\tilde{B}_{1}^{T} \mathcal{P}_{2} \tilde{B}_{2} ; \\
& \Upsilon_{8}=B_{2}^{T} P_{1} B_{2}+\tilde{B}_{2}^{T} \mathcal{P}_{2} \tilde{B}_{2}
\end{aligned}
$$

and the condition $\mathcal{P}_{2} \bar{K}=\mathcal{X}$ has been utilized.

By setting $\mathcal{W}=\operatorname{col}\left(\mathcal{W}_{1}, 0,0, \mathcal{W}_{4}\right)$ and

$$
\mathcal{W}_{1}=\left[\begin{array}{cc}
W_{111} & 0 \\
W_{121} & \mathcal{P}_{2}
\end{array}\right] \quad \mathcal{W}_{4}=\left[\begin{array}{cc}
W_{411} & 0 \\
W_{421} & -\mathcal{P}_{2}
\end{array}\right]
$$

one has

$$
\mathcal{W} \mathcal{T}=\left[\begin{array}{cccc}
-\mathcal{W}_{1} \mathcal{A} & -\mathcal{W}_{1} \mathcal{D} & -\mathcal{W}_{1} \mathcal{B}_{1} & \mathcal{W}_{1} \\
0 & 0 & 0 & 0 \\
0 & 0 & 0 & 0 \\
-\mathcal{W}_{4} \mathcal{A} & -\mathcal{W}_{4} \mathcal{D} & -\mathcal{W}_{4} \mathcal{B}_{1} & \mathcal{W}_{4}
\end{array}\right]
$$

where $\mathcal{T}$ is as defined in Theorem 1 and

$$
\begin{gathered}
\mathcal{W}_{1} \mathcal{A}=\left[\begin{array}{cc}
W_{111} A & 0 \\
W_{121} A & \mathcal{P}_{2}\left(I_{N} \otimes A\right)-\mathcal{X} \Gamma \tilde{C}
\end{array}\right] \\
\mathcal{W}_{1} \mathcal{D}=\left[\begin{array}{cc}
W_{111} D & 0 \\
W_{121} D+\mathcal{P}_{2} \tilde{D} & 0
\end{array}\right] \\
\mathcal{W}_{4} \mathcal{A}=\left[\begin{array}{cc}
W_{411} A & 0 \\
W_{421} A & -\mathcal{P}_{2}\left(I_{N} \otimes A\right)+\mathcal{X} \Gamma \tilde{C}
\end{array}\right] \\
\mathcal{W}_{4} \mathcal{D}=\left[\begin{array}{cc}
W_{411} D & 0 \\
W_{421} D-\mathcal{P}_{2} \tilde{D} & 0
\end{array}\right] \\
\mathcal{W}_{1} \mathcal{B}_{1}=\left[\begin{array}{cc}
\bar{\delta} W_{111} B_{1} & (1-\bar{\delta}) W_{111} B_{2} \\
\bar{\delta} W_{121} B_{1} & (1-\bar{\delta}) W_{121} B_{2} \\
0 & 0 \\
\bar{\delta} \mathcal{P}_{2}\left(I_{N} \otimes B_{1}\right) & (1-\bar{\delta}) \mathcal{P}_{2}\left(I_{N} \otimes B_{2}\right)
\end{array}\right] \\
\mathcal{W}_{4} \mathcal{B}_{1}=\left[\begin{array}{cc}
\bar{\delta} W_{411} B_{1} & (1-\bar{\delta}) W_{411} B_{2} \\
\bar{\delta} W_{421} B_{1} & (1-\bar{\delta}) W_{421} B_{2} \\
0 & 0 \\
-\bar{\delta} \mathcal{P}_{2}\left(I_{N} \otimes B_{1}\right) & -(1-\bar{\delta}) \mathcal{P}_{2}\left(I_{N} \otimes B_{2}\right)
\end{array}\right] .
\end{gathered}
$$

From the proof of Theorem 1 and the detailed matrices computed above, we obtain that the inequality $\Phi+\Theta\left(I_{2 N+1} \otimes\right.$ $\left.\mathcal{P}_{2}^{-1}\right) \Theta^{T}<0$ in (27) ensures the conditions in (15) to hold, which in turn illustrates that the network in (12) with timevarying delay is globally asymptotically stable in the mean square sense. From Definition 1, one knows that the system (6) is a convergent distributed state estimator of the networked dynamic system (1) with measurement outputs (2), and the proof is then completed.

Remark 4: In this paper, sufficient conditions are derived to ensure the system (6) to be a convergent distributed state estimator of the networked dynamic system (1) with measurement outputs (2). It should be noted that, by the Schur complement [38], the inequalities in (27) can be readily solved by using some standard numerical software, which further illustrates the practical usefulness of our results. On the other hand, by utilizing a method similar to that employed in [28], it is not difficult to deal with the robustness issues for the systems (1) and (2) with parameter uncertainties.

Remark 5: Distributed state estimation problem was also considered in [39] for wireless sensor network based on quantized observations. There are two main differences between 
the works in [39] and ours. 1) The networked dynamic target studied in [39] was a linear discrete-time system while the target investigated here is a delayed model with randomly occurred nonlinearities. 2) In [39], the authors first designed the quantized message function $m_{i}(k) \triangleq Q\left(y_{i}(k)\right)$ and the fusion function $g\left(m_{1}(k), m_{2}(k), \ldots, m_{N}(k)\right)$, and then constructed a linear filter to make the estimation error with a minimized upper bound, while in our work, veritable distributed state estimators are designed which could be reflected by the term $\sum_{j \in \mathcal{N}_{i}} l_{i j} K_{i j} y_{j}(k)$. Compared to the fusion function $g\left(m_{1}(k), m_{2}(k), \ldots, m_{N}(k)\right)$, the distributed estimators employed here are more practical. The reason can be explained just as pointing out in [34]: in a sensor network consisting of a large number of sensor nodes in a wide spatial region, it is impossible to have only one centralized processor that can collect the measurements from all the sensors, particularly in a remote area, while, usually, each sensor may only be able to use local information and communicate with neighbors so that the estimation can be achieved in a distributed way.

When the nonlinearities in the networked dynamic target are not randomly varying, i.e., the target system turns to be

$$
\begin{aligned}
x(k+1)= & A x(k)+D x(k-\tau(k)) \\
& +B f(k, x(k))+M_{0} x(k) \omega_{0}(k)
\end{aligned}
$$

we then design the following distributed state estimator:

$$
\begin{aligned}
\hat{x}_{i}(k+1)= & A \hat{x}_{i}(k)+B f\left(k, \hat{x}_{i}(k)\right) \\
& +\sum_{j \in \mathcal{N}_{i}} l_{i j} K_{i j}\left(y_{j}(k)-\bar{\gamma}_{j} C_{j} \hat{x}_{j}(k)\right) .
\end{aligned}
$$

Furthermore, if there is no time-varying delay in the target model, the target plant reduces to

$$
x(k+1)=A x(k)+B f(k, x(k))+M_{0} x(k) \omega_{0}(k) .
$$

Based on the analysis of Theorems 1 and 2, the above three special cases are easy to deal with and we have the following corollaries.

Corollary 1: Under Assumptions 1 and 2, the system (31) is a convergent distributed state estimator of the networked dynamic system (30) with measurement outputs (2) if there exist matrices $P_{j}>0(j=1,2, \ldots, N+1), Q_{11}>0, \mathcal{Q}_{22}>0$, matrices $Q_{12}, W_{i 11}, W_{i 21}(i=1,4), \mathcal{X} \in \mathscr{W}_{n \times m}$ and scalar $\varepsilon>0$ such that

$$
\mathcal{Q} \triangleq\left[\begin{array}{cc}
Q_{11} & Q_{12} \\
* & \mathcal{Q}_{22}
\end{array}\right]>0 \quad \Phi^{\prime}+\Theta\left(I_{2 N+1} \otimes \mathcal{P}_{2}^{-1}\right) \Theta^{T}<0
$$

where $\Phi^{\prime}$ can be derived from matrix $\Phi$ defined in Theorem 2 by firstly deleting the sixth and eighth rows and columns, and then substituting the number $\bar{\delta}$ and the matrices $B_{1}$ and $\Sigma_{2}$, respectively, by the number 1 and the matrices $B$ and 0 , the other symbols are the same as defined in Theorem 2 . Moreover, $\bar{K}=\mathcal{P}_{2}^{-1} \mathcal{X}$, accordingly, the state estimator gains $K_{i j}\left(i=1,2, \ldots, N, j \in \mathcal{N}_{i}\right)$ can be derived from (9).

Corollary 2: Under Assumptions 1 and 2, the system (31) is a convergent distributed state estimator of the networked dynamic system (32) with measurement outputs (2) if there exist matrices $P_{j}>0(j=1,2, \ldots, N+1)$, matrices $W_{i 11}$, $W_{i 21}(i=1,4), \mathcal{X} \in \mathscr{W}_{n \times m}$, and scalar $\varepsilon>0$ such that

$$
\Phi^{\prime \prime}+\Theta\left(I_{2 N+1} \otimes \mathcal{P}_{2}^{-1}\right) \Theta^{T}<0
$$

where $\Phi^{\prime \prime}$ can be derived from matrix $\Phi^{\prime}$ defined in Corollary 1 by first deleting the third and fourth rows and columns, and then substituting the matrices $Q_{11}, Q_{12}$, and $\mathcal{Q}_{22}$ all by the zero matrix 0 with appropriate dimensions, the other symbols are the same as defined in Theorem 2. Moreover, $\bar{K}=\mathcal{P}_{2}^{-1} \mathcal{X}$, accordingly, the state estimator gains $K_{i j}(i=$ $1,2, \ldots, N, j \in \mathcal{N}_{i}$ ) can be derived from (9).

Remark 6: Assume that there are neither randomly varying nonlinearities nor stochastic disturbances in the target plant (1) and in the corresponding sensor networks (2). Assume also that there are no probabilistic missing data probabilities, i.e., the sensor network measurements are the ideal (perfect) outputs where $\gamma_{i}(k) \equiv \bar{\gamma}_{i}=1$. In this case, the model reduces to

$$
x(k+1)=A x(k)+D x(k-\tau(k))+B f(k, x(k))
$$

and

$$
y_{i}(k)=C_{i} x(k), \quad i=1,2, \ldots, N .
$$

Considering the target plant (35) with $N$ sensors modeled by (36), we further implement the following full-order distributed state estimator:

$$
\begin{aligned}
\hat{x}_{i}(k+1)= & A \hat{x}_{i}(k)+D \hat{x}_{i}(k-\tau(k))+B f\left(k, \hat{x}_{i}(k)\right) \\
& +\sum_{j \in \mathcal{N}_{i}} l_{i j} K_{i j}\left(y_{j}(k)-C_{j} \hat{x}_{j}(k)\right)
\end{aligned}
$$

and then the error state vector $\tilde{x}(k)=\left(\tilde{x}_{1}^{T}(k), \tilde{x}_{2}^{T}(k), \ldots\right.$, $\left.\tilde{x}_{N}^{T}(k)\right)^{T}$ with $\tilde{x}_{i}(k)=x(k)-\hat{x}_{i}(k)$ satisfies the following equation:

$$
\begin{aligned}
\tilde{x}(k+1)= & \left(I_{N} \otimes A-\bar{K} \tilde{C}\right) \tilde{x}(k)+\left(I_{N} \otimes D\right) \tilde{x}(k-\tau(k)) \\
& +\left(I_{N} \otimes B\right) F(k, \tilde{x}(k)) .
\end{aligned}
$$

Along similar lines as the proof of Theorem 1, we can obtain some sufficient conditions that guarantee the system (37) to be a convergent distributed state estimator of the dynamic system (35) with measurement outputs (36), and these sufficient conditions will no longer require the original system (35) to be asymptotically stable.

Remark 7: The algorithm developed in this paper is based on the linear matrix inequalities (LMIs) that can be solved by MATLAB LMI toolbox. This algorithm has a polynomial-time complexity, which is bounded by $O\left(v_{1} v_{2} 3 \log (\vartheta / \epsilon)\right)$, where $v_{1}$ is the total row size of the LMI system, $v_{2}$ is the total number of scalar decision variables, $\vartheta$ is a data-dependent scaling factor, and $\epsilon$ is the relative accuracy set for the algorithm. For example, let us examine the distributed state estimator (6) for the systems (1) and (2), the dimensions of system variables are $x(k) \in \mathbb{R}^{n}$ and $y_{i}(k) \in \mathbb{R}^{m}(i=1,2, \ldots, N)$. From Theorem 2, we have $v_{1}=6 n+7 n N+2 n N^{2}$ and $v_{2}=n^{2}\left(3+(7 / 2) N+(1 / 2) N^{2}\right)+m n N^{2}+n N+n+1$, therefore the time complexity of our algorithm can be represented as $O\left(n^{3} N^{4}\right)$, which is obviously dependent on the number of sensors $N$ and the number of target plant states $n$. On the other hand, the MATLAB LMI Toolbox implements stateof-the-art interior-point LMI solvers. While these solvers are significantly faster than classical convex optimization algorithms, it should be kept in mind that the complexity of LMI computations remains higher than that of solving, say, a 


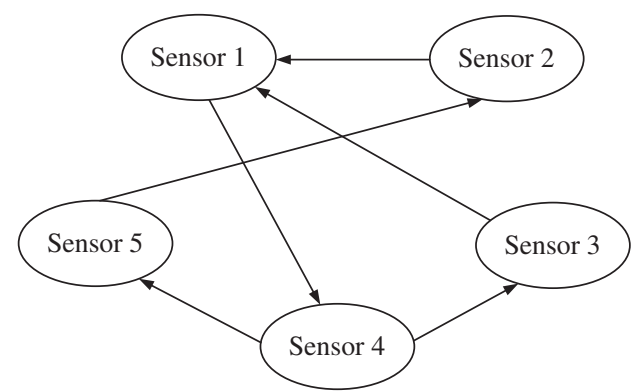

Fig. 1. Topological structure of the sensor network.

Riccati equation. For instance, problems with a thousand design variables typically take over an hour on today's workstations. However, research on LMI optimization is a very active area in the applied mathematics, optimization, and the operations research community, and substantial speedups can be expected in the future.

\section{NUMERICAL EXAMPLES}

Consider a networked target system (1) with the following parameters:

$$
\begin{aligned}
A & =\left[\begin{array}{ccc}
-0.1 & 0.01 & 0 \\
0 & 0.07 & -0.01 \\
-0.01 & 0 & 0.06
\end{array}\right] \\
D & =\left[\begin{array}{ccc}
-0.1 & 0.1 & 0.1 \\
0.2 & 0.1 & 0 \\
-0.1 & -0.2 & 0.1
\end{array}\right] \\
B_{1} & =\left[\begin{array}{ccc}
0.2 & -0.1 & 0 \\
0.1 & -0.1 & 0 \\
0 & -0.2 & -0.1
\end{array}\right] \\
B_{2} & =\left[\begin{array}{ccc}
-0.2 & 0 & 0.1 \\
-0.2 & -0.1 & 0.1 \\
0 & 0.2 & -0.1
\end{array}\right] \\
M_{0} & =\left[\begin{array}{ccc}
0.2 & -0.1 & 0.1 \\
0.5 & -0.5 & 0 \\
0.3 & -0.1 & 0.2
\end{array}\right] .
\end{aligned}
$$

The time-varying delay in (1) is assumed to be $\tau(k)=$ $2+3|\sin ((k / 2) \pi)|$, i.e., the delay $\tau(k)$ has upper bound $\tau_{2}=5$ and lower bound $\tau_{1}=2$. The randomly occurred nonlinearities are

$$
\begin{aligned}
f(k, x(k))= & \left(0.1 x_{1}(k)-\tanh \left(0.2 x_{1}(k)\right),\right. \\
& \left.0.1 x_{2}(k)-\tanh \left(0.1 x_{2}(k)\right), 0.2 x_{3}(k)\right)^{T}
\end{aligned}
$$

and

$$
\begin{aligned}
g(k, x(k))= & \left(-0.2 x_{1}(k)\right), \\
& \left.0.2 x_{2}(k)-\tanh \left(0.1 x_{2}(k)\right), \tanh \left(0.1 x_{3}(k)\right)\right)^{T}
\end{aligned}
$$

i.e., they satisfy Assumptions 1 and 2 with $\Sigma_{1}=$ $\operatorname{diag}(0.3,0.2,0.2)$ and $\Sigma_{2}=\operatorname{diag}(0.2,0.3,0.1)$.

The sensor network has five nodes with interconnection topology shown in Fig. 1, i.e., the adjacency matrix

$$
L=\left[\begin{array}{lllll}
1 & 1 & 1 & 0 & 0 \\
0 & 1 & 0 & 0 & 1 \\
0 & 0 & 1 & 1 & 0 \\
1 & 0 & 0 & 1 & 0 \\
0 & 0 & 0 & 1 & 1
\end{array}\right]
$$

The dynamics of the sensor nodes are described by (2) with parameters as follows:

$$
\begin{aligned}
C_{1} & =\left[\begin{array}{ccc}
0 & -0.1 & 0.1 \\
0.1 & 0.2 & 0.3
\end{array}\right] \\
C_{2} & =\left[\begin{array}{ccc}
0.3 & 0 & 0.1 \\
0 & 0 & 0
\end{array}\right] \\
C_{3} & =\left[\begin{array}{ccc}
0.1 & 0.2 & -0.2 \\
0.2 & -0.1 & 0.1
\end{array}\right] \\
C_{4} & =\left[\begin{array}{ccc}
0 & 0.1 & -0.2 \\
-0.1 & 0.2 & 0
\end{array}\right] \\
C_{5} & =\left[\begin{array}{ccc}
0.1 & 0.3 & 0.4 \\
0.3 & -0.2 & 0.1
\end{array}\right] \\
M_{1} & =\left[\begin{array}{ccc}
0.4 & 0.1 & -0.1 \\
0 & -0.1 & 0.3
\end{array}\right] \\
M_{2} & =\left[\begin{array}{ccc}
0.1 & 0.1 & 0 \\
0.3 & 0.2 & -0.2
\end{array}\right] \\
M_{3} & =\left[\begin{array}{ccc}
-0.1 & 0.1 & 0 \\
0 & -0.1 & 0.2
\end{array}\right] \\
M_{4} & =\left[\begin{array}{ccc}
0.1 & -0.2 & 0 \\
-0.1 & 0.1 & 0.2
\end{array}\right] \\
M_{5} & =\left[\begin{array}{ccc}
0.2 & -0.3 & 0.1 \\
-0.1 & 0.2 & 0
\end{array}\right] .
\end{aligned}
$$

And the Bernoulli-distributed white noise sequences $\delta(k)$ and $\gamma_{i}(k)(i=1,2,3,4,5)$ are assumed to satisfy conditions (4) and (5) with

$$
\begin{gathered}
\bar{\delta}=0.68, \quad \bar{\gamma}_{1}=0.15, \quad \bar{\gamma}_{2}=0.86, \\
\bar{\gamma}_{3}=0.96, \quad \bar{\gamma}_{4}=0.26, \quad \bar{\gamma}_{5}=0.76 .
\end{gathered}
$$

By resorting to some standard software in MATLAB, the matrix inequalities in (27) are solvable with a feasible solution as follows (here only part of the matrices are listed for simplicity): $\varepsilon=6.6613$

$$
\begin{aligned}
P_{1} & =\left[\begin{array}{ccc}
77.6371 & -18.5079 & -7.1468 \\
-18.5079 & 63.5472 & 0.2328 \\
-7.1468 & 0.2328 & 24.5598
\end{array}\right] \\
Q_{11} & =\left[\begin{array}{ccc}
10.1110 & 2.0258 & -2.9191 \\
2.0258 & 8.5853 & 0.4305 \\
-2.9191 & 0.4305 & 4.2383
\end{array}\right] \\
W_{111} & =\left[\begin{array}{ccc}
-0.6846 & -0.0799 & -0.0074 \\
0.0818 & 0.5333 & -0.3403 \\
-0.0653 & -0.1722 & 0.3198
\end{array}\right] \\
W_{411} & =\left[\begin{array}{ccc}
-3.4720 & 0.0648 & -0.2055 \\
0.0761 & -3.5230 & -0.0818 \\
0.2021 & -0.0988 & -3.4878
\end{array}\right] .
\end{aligned}
$$


Therefore, it follows from Theorem 2 that the system (6) is a convergent distributed state estimator of the networked dynamic system (1) with measurement outputs (2). Furthermore, by (9) and (28), we can derive the distributed state estimator gain matrices in (6) to be

$$
\begin{aligned}
& K_{11}=\left[\begin{array}{cc}
-0.0762 & -0.2710 \\
0.0752 & -0.1040 \\
-0.2016 & -0.3428
\end{array}\right] \\
& K_{12}=\left[\begin{array}{cc}
-0.0492 & 0.0153 \\
0.0237 & -0.0073 \\
-0.0251 & 0.0078
\end{array}\right] \\
& K_{13}=\left[\begin{array}{cc}
-0.0275 & -0.0779 \\
-0.0571 & 0.0559 \\
0.0760 & -0.0424
\end{array}\right] \\
& K_{22}=\left[\begin{array}{ll}
-1.1194 & 0.3542 \\
-0.0058 & 0.0020 \\
-0.2156 & 0.0683
\end{array}\right] \\
& K_{25}=\left[\begin{array}{cc}
0.0031 & -0.0541 \\
-0.0096 & 0.0618 \\
-0.0303 & -0.0436
\end{array}\right] \\
& K_{33}=\left[\begin{array}{cc}
-0.6291 & -1.1389 \\
-0.3707 & 0.1318 \\
0.3451 & -0.1952
\end{array}\right] \\
& K_{34}=\left[\begin{array}{cc}
0.0086 & 0.1122 \\
-0.1971 & -0.1735 \\
0.2692 & 0.1055
\end{array}\right] \\
& K_{41}=\left[\begin{array}{cc}
-0.0127 & -0.0371 \\
0.0591 & 0.0344 \\
-0.0894 & -0.1044
\end{array}\right] \\
& K_{44}=\left[\begin{array}{cc}
0.1112 & 0.7149 \\
-0.4497 & -0.5894 \\
0.6637 & 0.2091
\end{array}\right] \\
& K_{54}=\left[\begin{array}{cc}
-0.0071 & 0.1104 \\
-0.2090 & -0.1730 \\
0.2751 & 0.1086
\end{array}\right] \\
& K_{55}=\left[\begin{array}{cc}
-0.1996 & -0.8298 \\
-0.1817 & 0.2227 \\
-0.2707 & -0.2144
\end{array}\right] \text {. }
\end{aligned}
$$

\section{CONCLUSion AND Discussions}

In this paper, the problem of distributed state estimation was investigated for sensor networks with time-varying delay and randomly varying nonlinearities in discrete-time form. The sensor network consisted of $N$ nodes, and the dynamics of each sensor was characterized by a linear model with missing measurement. To reflect more realistic situations, stochastic disturbances were considered in both the target model and the sensor dynamics. Distributed state estimators were designed to estimate the states of the target plant through the sensor measurements in a distributed way. By employing the Lyapunov stability theory and the stochastic analysis technique, it was verified that such estimators exist if a set of matrix inequalities are solvable. Furthermore, the effectiveness of these criteria were checked by a numerical example.

We like to point out that our main results can be extended to other uncertain discrete-time dynamical systems such as those with Markovian jumping parameters and/or distributed time-delays. The sensor network discussed in this paper is currently represented by a direct graph. If the topology is described by an undirected graph, i.e., the nonnegative adjacency matrix $L=\left(l_{i j}\right)_{N \times N}$ is symmetric, then the designed distributed state estimators will have a better performance for the state estimation. This will also be one of our future research topics.

\section{REFERENCES}

[1] J. Cao, G. Chen, and P. Li, "Global synchronization in an array of delayed neural networks with hybrid coupling," IEEE Trans. Syst., Man, Cybern., Part B, vol. 38, no. 2, pp. 488-498, Apr. 2008.

[2] Y. Liu, Z. Wang, J. Liang, and X. Liu, "Synchronization and state estimation for discrete-time complex networks with distributed delays," IEEE Trans. Syst., Man Cybern., Part B, vol. 38, no. 5, pp. 1314-1325, Oct. 2008.

[3] W. Lu and T. Chen, "Synchronisation in complex networks of coupled systems with directed topologies," Int. J. Syst. Sci., vol. 40, no. 9, pp. 909-921, Sep. 2009.

[4] S. Mou, H. Gao, W. Qiang, and K. Chen, "New delay-dependent exponential stability for neural networks with time delay," IEEE Trans. Syst., Man, Cybern., Part B, vol. 38, no. 2, pp. 571-576, Apr. 2008.

[5] S. Mou, Y. Zhao, H. Gao, and W. Qiang, "Further improvement on synchronization stability of complex networks with coupling delays," Int. J. Comput. Math., vol. 85, no. 8, pp. 1255-1263, Aug. 2008.

[6] Y. Tang, S. Y. S. Leung, W. K. Wong, and J. Fang, "Impulsive pinning synchronization of stochastic discrete-time networks," Neurocomputing, vol. 73, nos. 10-12, pp. 2132-2139, Jun. 2010.

[7] X. F. Wang and G. Chen, "Synchronization in scale-free dynamical networks: Robustness and fragility," IEEE Trans. Circuits Syst. I: Fundam. Theory Appl., vol. 49, no. 1, pp. 54-62, Jan. 2002.

[8] R. R. Brooks, P. Ramanathan, and A. M. Sayeed, "Distributed target classification and tracking in sensor networks," Proc. IEEE, vol. 91, no. 8, pp. 1163-1171, Aug. 2003.

[9] Z. Xiong, A. D. Liveris, and S. Cheng, "Distributed source coding for sensor networks," IEEE Signal Process. Mag., vol. 21, no. 5, pp. 80-94, Sep. 2004.

[10] H. Gao and T. Chen, " $H_{\infty}$ estimation for uncertain systems with limited communication capacity," IEEE Trans. Autom. Control, vol. 52, no. 11, pp. 2070-2084, Nov. 2007.

[11] H. Liu, F. Sun, K. He, and Z. Sun, "Design of reduced-order $\mathcal{H}_{\infty}$ filter for Markovian jumping systems with time delay," IEEE Trans. Circuits Syst. II, vol. 51, no. 11, pp. 607-612, Nov. 2004.

[12] P. Shi, M. Mahmoud, S. K. Nguang, and A. Ismail, "Robust filtering for jumping systems with mode-dependent delays," Signal Process., vol. 86 , no. 1, pp. 140-152, Jan. 2006.

[13] L. Wu, P. Shi, H. Gao, and C. Wang, "H户 $\mathcal{H}_{\infty}$ filtering for 2-D Markovian jump systems," Automatica, vol. 44, no. 7, pp. 1849-1858, Jul. 2008.

[14] J. Xiong and J. Lam, "Fixed-order robust $\mathcal{H}_{\infty}$ filter design for Markovian jump systems with uncertain switching probabilities," IEEE Trans. Signal Process., vol. 54, no. 4, pp. 1421-1430, Apr. 2006.

[15] H. Zhang, G. Feng, G. Duan, and X. Lu, "H户 $\mathcal{H}_{\infty}$ filtering for multipletime-delay measurements," IEEE Trans. Signal Process., vol. 54, no. 5, pp. 1681-1688, May 2006.

[16] P. Alriksson and A. Rantzer, "Distributed Kalman filtering using weighted averaging," in Proc. 17th Int. Symp. Math. Theory Netw. Syst., Kyoto, Japan, Jul. 2006.

[17] R. Carli, A. Chiuso, L. Schenato, and S. Zampieri, "Distributed Kalman filtering based on consensus strategies," IEEE J. Sel. Areas Commun., vol. 26, no. 4, pp. 622-633, May 2008.

[18] M. Kamgarpour and C. Tomlin, "Convergence properties of a decentralized Kalman filter," in Proc. 47th IEEE Conf. Decis. Control, Cancun, Mexico, Dec. 2008, pp. 3205-3210. 
[19] R. Olfati-Saber, "Distributed Kalman filtering for sensor networks," in Proc. 46th IEEE Conf. Decis. Control, New Orleans, LA, 2007, pp. $1-7$.

[20] R. Olfati-Saber and J. S. Shamma, "Consensus filters for sensor networks and distributed sensor fusion," in Proc. 44th IEEE Conf. Decis. Control, Eur. Control Conf., Seville, Spain, Dec. 2005, pp. 6698-6703.

[21] D. P. Spanos, R. Olfati-Saber, and R. M. Murray, "Approximate distributed Kalman filtering in sensor networks with quantifiable performance," in Proc. 4th Int. Symp. Inf. Process. Sens. Netw., Apr. 2005, pp. 133-139.

[22] M. Farina, G. Ferrari-Trecate, and R. Scattolini, "Distributed moving horizon estimation for sensor networks," in Proc. 1st IFAC Workshop Estim. Control Netw. Syst., Venice, Italy, 2009, pp. 126-131.

[23] M. Farina, G. Ferrari-Trecate, and R. Scattolini, "A moving horizon scheme for distributed state estimation," in Proc. 48th IEEE Conf. Decis. Control, Shanghai, China, Dec. 2009, pp. 1818-1823.

[24] A. Speranzon, C. Fischione, K. H. Johansson, and A. SangiovanniVincentelli, "A distributed minimum variance estimator for sensor networks," IEEE J. Sel. Areas Commun., vol. 26, no. 4, pp. 609-621, May 2008.

[25] J.-H. Kim, M. West, E. Scholte, and S. Narayanan, "Multiscale consensus for decentralized estimation and its application to building systems," in Proc. Amer. Control Conf., Seattle, WA, Jun. 2008, pp. 888-893.

[26] F. S. Cattivelli and A. H. Sayed, "Diffusion LMS strategies for distributed estimation,” IEEE Trans. Signal Process., vol. 58, no. 3, pp. 10351048, Mar. 2010.

[27] F. S. Cattivelli and A. H. Sayed, "Diffusion strategies for distributed Kalman filtering and smoothing," IEEE Trans. Autom. Control, vol. 55, no. 9, pp. 2069-2084, Sep. 2010.

[28] J. Liang, Z. Wang, and X. Liu, "State estimation for coupled uncertain stochastic networks with missing measurements and time-varying delays: The discrete-time case," IEEE Trans. Neural Netw., vol. 20, no. 5, pp. 781-793, May 2009.

[29] B. Sinopoli, L. Schenato, M. Franceschetti, K. Poolla, M. I. Jordan, and S. S. Sastry, "Kalman filtering with intermittent observations," IEEE Trans. Autom. Control, vol. 49, no. 9, pp. 1453-1464, Sep. 2004.

[30] Z. Wang, D. W. C. Ho, Y. Liu, and X. Liu, "Robust $H_{\infty}$ control for a class of nonlinear discrete time-delay stochastic systems with missing measurements," Automatica, vol. 45, no. 3, pp. 684-691, Mar. 2009.

[31] Y. Wang, Z. Wang, and J. Liang, "Global synchronization for delayed complex networks with randomly occurring nonlinearities and multiple stochastic disturbances," J. Phys. A: Math. Theor, vol. 42, no. 13, pp. 135101-1-135101-19, Apr. 2009.

[32] Z. Wang, Y. Wang, and Y. Liu, "Global synchronization for discrete-time stochastic complex networks with randomly occurred nonlinearities and mixed time delays," IEEE Trans. Neural Netw., vol. 21, no. 1, pp. 11-25, Jan. 2010

[33] C. Lin, Z. Wang, and F. Yang, "Observer-based networked control for continuous-time systems with random sensor delays," Automatica, vol. 45, no. 2, pp. 578-584, Feb. 2009.

[34] W. Yu, G. Chen, Z. Wang, and W. Yang, "Distributed consensus filtering in sensor networks," IEEE Trans. Syst., Man, Cybern, Part B, vol. 39, no. 6, pp. 1568-1577, Dec. 2009.

[35] F. Yang, Z. Wang, and D. W. C. Ho, "Robust mixed $H_{2} / H_{\infty}$ control for a class of nonlinear stochastic systems," IEE Proc., Control Theory Appl., vol. 153, no. 2, pp. 175-184, Mar. 2006.

[36] N. Nahi, "Optimal recursive estimation with uncertain observation," IEEE Trans. Inf. Theory, vol. 15, no. 4, pp. 457-462, Jul. 1969.

[37] H. Gao, J. Lam, and Z. Wang, "Discrete bilinear stochastic systems with time-varying delay: Stability analysis and control synthesis," Chaos, Solitons Fractals, vol. 34, no. 2, pp. 394-404, Oct. 2007.

[38] S. Boyd, L. E. Ghaoui, E. Feron, and V. Balakrishnan, Linear Matrix Inequalities in System and Control Theory. Philadelphia, PA: SIAM, 1994.

[39] L. Chai, B. Hu, and P. Jiang, "Distributed state estimation based on quantized observations in a bandwidth constrained sensor network," in Proc. 7th World Congr. Intell. Control Autom., Chongqing, China, Jun. 2008, pp. 2411-2415.

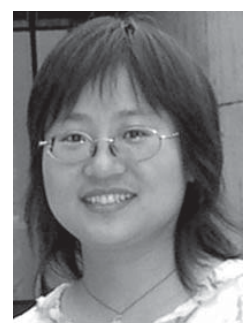

Jinling Liang received the B.Sc. and M.Sc. degrees in mathematics from Northwest University, Xian, China, in 1997 and 1999, respectively, and the $\mathrm{Ph} . \mathrm{D}$. degree in applied mathematics from Southeast University, Nanjing, China, in 2006.

She was a Research Associate at the University of Hong Kong, Hong Kong, from January 2004 to March 2004. From March to April 2004, she was a Research Associate at the City University of Hong Kong, Hong Kong. From April 2007 to March 2008, she was a Post-Doctoral Research Fellow (sponsored by the Royal Society Sino-British Fellowship Trust Award of U.K.) and a Visiting Research Fellow (sponsored by the Royal Society of U.K.) in the Department of Information Systems and Computing, Brunel University, Uxbridge, U.K., from January to March 2010. She is currently an Associate Professor in the Department of Mathematics, Southeast University. She has published around 20 papers in refereed international journals. Her current research interests include neural networks, complex networks, nonlinear systems, and bioinformatics.

Dr. Liang is a member of the program committees of several international conferences and serves as a very active reviewer for many international journals.

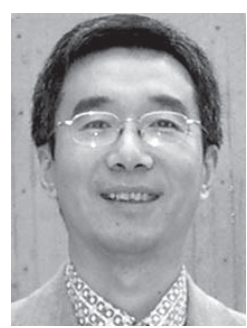

Zidong Wang (SM'03) was born in Jiangsu, China, in 1966. He received the B.Sc. degree in mathematics from Suzhou University, Suzhou, China, in 1986, the M.Sc. degree in applied mathematics and the Ph.D. degree in electrical engineering both from Nanjing University of Science and Technology, Nanjing, China, in 1990 and 1994, respectively.

He held teaching and research appointments in universities in China, Germany, and U.K., from 1990 to 2002. He is currently a Professor of dynamical systems and computing in the Department of Information Systems and Computing, Brunel University, Uxbridge, U.K. $\mathrm{He}$ has published more than 100 papers in refereed international journals. His current research interests include dynamical systems, signal processing, bioinformatics, and control theory and applications.

Prof. Wang serves as an Associate Editor for 11 international journals, including the IEEE TRANSACTIONS ON Automatic CONTROL, the IEEE TRANSACTIONS ON CONTROL SYSTEMS TECHNOLOGY, the IEEE TRANSaCtions on NeUral Networks, the IEEE TransaCtions on Signal Processing, and the IEEE TRANSACTIONS ON SYSTEMS, MAN, AND CYBERNETICS-PART C. He has been a member of the program committees of many international conferences. He is a Fellow of the Royal Statistical Society and is a recipient of the Alexander von Humboldt Research Fellowship, Germany, the Japan Society for the Promotion of Science Research Fellowship, Japan, and the William Mong Visiting Research Fellowship, Hong Kong.

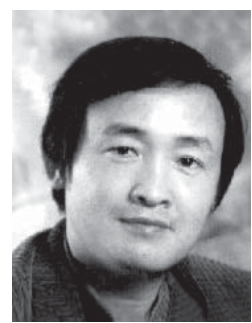

Xiaohui Liu received the B.Eng. degree in computing from Hohai University, Nanjing, China, in 1982, and the Ph.D. degree in computer science from Heriot-Watt University, Edinburg, U.K., in 1988.

He is currently a Professor of computing at Brunel University, Uxbridge, U.K. He leads the Intelligent Data Analysis (IDA) Group. His current research interests include artificial intelligence, dynamic systems, image and signal processing, and statistics, particularly for applications in biology, engineering, and medicine.

Prof. Liu serves on the editorial boards of four computing journals, founded the Biennial International Conference series on IDA in 1995, and has given numerous invited talks in bioinformatics, data mining, and statistics conferences. 